\title{
A unified algorithm for solving split generalized mixed equilibrium problem, and for finding fixed point of nonspreading mapping in Hilbert spaces
}

https://doi.org/10.1515/dema-2018-0015

Received March 26, 2018; accepted June 15, 2018

\begin{abstract}
The purpose of this paper is to study a split generalized mixed equilibrium problem and a fixed point problem for nonspreading mappings in real Hilbert spaces. We introduce a new iterative algorithm and prove its strong convergence for approximating a common solution of a split generalized mixed equilibrium problem and a fixed point problem for nonspreading mappings in real Hilbert spaces. Our algorithm is developed by combining a modified accelerated Mann algorithm and a viscosity approximation method to obtain a new faster iterative algorithm for finding a common solution of these problems in real Hilbert spaces. Also, our algorithm does not require any prior knowledge of the bounded linear operator norm. We further give a numerical example to show the efficiency and consistency of our algorithm. Our result improves and compliments many recent results previously obtained in this direction in the literature.
\end{abstract}

Keywords: split mixed equilibrium, nonspreading mapping, fixed point problem, accelerated algorithm, iterative method, viscosity approximation method

MSC: 65K15, 47J25, 65J15, 90C33

\section{Introduction}

Let $H$ be a Hilbert space with the inner product $\langle\cdot, \cdot\rangle$ and the induced norm $\|\cdot\|$. Let $C$ be a nonempty, closed and convex subset of $H, \Theta: C \times C \rightarrow \mathbb{R}$ be a nonlinear bifunction, $h: C \rightarrow H$ be a nonlinear mapping, and $\phi: C \rightarrow \mathbb{R} \cup\{+\infty\}$ be a proper convex lower semicontinuous function. The Generalized Mixed Equilibrium Problem (GMEP) is defined as finding a point $x \in C$ such that

$$
\Theta(x, y)+\langle h x, y-x\rangle+\phi(y)-\phi(x) \geq 0, \quad \forall y \in C .
$$

The set of solutions of (1) is denoted by $\operatorname{GMEP}(\Theta, h, \phi)$.

If $h=0$, Problem (1) reduces to the Mixed Equilibrium Problem (MEP) which is to find a point $x \in C$ such that

\footnotetext{
Lateef Olakunle Jolaoso: School of Mathematics, Statistics and Computer Science, University of KwaZulu-Natal, Durban, South Africa; E-mail: 216074984@stu.ukzn.ac.za

Kazeem Olawale Oyewole: School of Mathematics, Statistics and Computer Science, University of KwaZulu-Natal, Durban, South Africa; Email: 217079141@stu.ukzn.ac.za

Chibueze Christian Okeke: School of Mathematics, Statistics and Computer Science, University of KwaZulu-Natal, Durban, South Africa; Email: 215082178@stu.ukzn.ac.za

*Corresponding Author: Oluwatosin Temitope Mewomo: School of Mathematics, Statistics and Computer Science, University of KwaZulu-Natal, Durban, South Africa; Email mewomoo@ukzn.ac.za
} 


$$
\Theta(x, y)+\phi(y)-\phi(x) \geq 0, \quad \forall y \in C .
$$

The set of solutions of (2) is denoted by $\operatorname{MEP}(\Theta, \phi)$.

In particular, if $\phi=0$ in (2), the MEP reduces to the classical equilibrium problem which was introduced by Blum and Oettli [1] and defined as finding a point $x \in C$ such that

$$
\Theta(x, y) \geq 0, \quad \forall y \in C .
$$

The GMEP is very general in the sense that it includes as special cases, minimization problems, variational inequality problems, fixed point problems, Nash equilibrium problems in noncooperative games and many others, see [2-4].

In 1994, Censor and Elfving [5] introduced the following Split Feasibility Problem (SFP) in finite-dimensional Hilbert spaces. Let $H_{1}, H_{2}$ be two Hilbert spaces, $C$ and $Q$ be nonempty closed convex subsets of $H_{1}$ and $H_{2}$ respectively, and let $A: H_{1} \rightarrow H_{2}$ be a bounded linear operator. The SFP is formulated as finding a point $x^{\dagger}$ with the property

$$
\chi^{\dagger} \in C \text { and } A x^{\dagger} \in Q
$$

The SFP has been applied extensively in many areas of science and engineering such as in intensitymodulated radiation therapy, signal processing and image reconstruction. The SFP has received attention from many authors, and various iterative methods have been proposed for finding its solutions, see for instance [6-9].

Let $H_{1}, H_{2}$ be real Hilbert spaces and let $C$ and $Q$ be nonempty closed convex subsets of $H_{1}$ and $H_{2}$, respectively. Let $\Theta_{1}: C \times C \rightarrow \mathbb{R}$ and $\Theta_{2}: Q \times Q \rightarrow \mathbb{R}$ be nonlinear bifunctions, $h_{1}: C \rightarrow H_{1}$ and $h_{2}: Q \rightarrow H_{2}$ be nonlinear mappings, $\phi: C \rightarrow \mathbb{R} \cup\{+\infty\}$ and $\varphi: Q \rightarrow \mathbb{R} \cup\{+\infty\}$ be proper lower semicontinuous and convex functions, and let $A: H_{1} \rightarrow H_{2}$ be a bounded linear operator. In this paper, we study the following Split Generalized Mixed Equilibrium Problem (SGMEP). Find a point $x^{\dagger} \in C$ such that

$$
\left\{\begin{array}{l}
\Theta_{1}\left(x^{\dagger}, x\right)+\left\langle h_{1}\left(x^{\dagger}\right), x-x^{\dagger}\right\rangle+\phi(x)-\phi\left(x^{\dagger}\right) \geq 0, \quad \forall x \in C, \\
\text { where } \\
y^{\dagger}=A x^{\dagger} \quad \text { solves } \Theta_{2}\left(y^{\dagger}, y\right)+\left\langle h_{2}\left(y^{\dagger}\right), y-y^{\dagger}\right\rangle+\varphi(y)-\varphi\left(y^{\dagger}\right) \geq 0, \quad \forall y \in Q .
\end{array}\right.
$$

The set of solutions of the SMEP is denoted by $\Omega:=\left\{x^{\dagger} \in G M E P\left(\Theta_{1}, h_{1}, \phi\right): A x^{\dagger} \in G M E P\left(\Theta_{2}, h_{2}, \varphi\right)\right\}$. We present the following examples to show that $\Omega$ is nonempty.

Example 1.1. Let $H_{1}=H_{2}=\mathbb{R}, C=[2, \infty)$ and $Q=(-\infty,-4]$. Let $A(x)=-2 x$ for all $x \in \mathbb{R}$, so that $A$ is a bounded linear operator. Let $\Theta_{1}: C \times C \rightarrow \mathbb{R}$ and $\Theta_{2}: Q \times Q \rightarrow \mathbb{R}$ be defined by $\Theta_{1}(x, y)=y-x$ and $\Theta_{2}(u, v)=3(u-v)$. Next, let $h_{1}: C \rightarrow \mathbb{R}$ and $h_{2}: Q \rightarrow \mathbb{R}$ be defined by $h_{1}(x)=x$ and $h_{2}(u)=2 u$. Finally, let $\phi: C \rightarrow \mathbb{R} \cup\{+\infty\}$ and $\varphi: Q \rightarrow \mathbb{R} \cup\{+\infty\}$ be defined by $\phi(x)=\frac{x^{2}}{2}$ and $\varphi(u)=2 u$. Clearly, $G M E P\left(\Theta_{1}, h, \phi\right)=$ $\{2\}$ and $A(2)=-4 \in G M E P\left(\Theta_{2}, h_{2}, \varphi\right)$. Thus, $\Omega=\left\{p \in G M E P\left(\Theta_{1}, h_{1}, \phi\right): A p \in G M E P\left(\Theta_{2}, h_{2}, \varphi\right)\right\} \neq \emptyset$.

Example 1.2. Let $H_{1}=\mathbb{R}^{2}$ with the norm $\|\bar{x}\|=\sqrt{x_{1}^{2}+x_{2}^{2}}$ for $\bar{x}=\left(x_{1}, x_{2}\right) \in \mathbb{R}^{2}$, and let $H_{2}=\mathbb{R}$. Let $C:=\left\{\bar{x}=\left(x_{1}, x_{2}\right) \in \mathbb{R}^{2}: x_{2}-x_{1} \geq 1\right\}$ and $Q=[1, \infty)$. Define $\Theta_{1}(\bar{x}, \bar{y})=y_{2}-y_{1}-x_{2}+x_{1}$, where $\bar{x}=\left(x_{1}, x_{2}\right)$ and $\bar{y}=\left(y_{1}, y_{2}\right) \in C$; then $\Theta_{1}$ is a bifunction from $C \times C \rightarrow \mathbb{R}$. Let $h_{1}(\bar{x})=\phi(\bar{x})=x_{2}-x_{1}$, then $G M E P\left(\Theta_{1}, h_{1}, \phi\right)=$ $\left\{\bar{q}=\left(q_{1}, q_{2}\right): q_{2}-q_{1}=1\right\}$. Also define $\Theta_{2}(u, v)=v-u$ for all $u, v \in Q$, so that $\Theta_{2}$ is a bifunction from $Q \times Q$ to $\mathbb{R}$, and let $h_{2}(u)=2 u, \varphi(u)=u$. For each $\bar{x}=\left(x_{1}, x_{2}\right) \in H_{1}$, let $A(\bar{x})=x_{2}-x_{1}$, so that $A$ is bounded linear operator from $H_{1}$ into $H_{2}$. Clearly, when $\bar{q} \in G M E P\left(\Theta_{1}, h_{1}, \phi\right)$, we have $A \bar{q}=1 \in G M E P\left(\Theta_{2}, h_{2}, \varphi\right)$. Thus $\Omega=\left\{\bar{q} \in \operatorname{GMEP}\left(\Theta_{1}, h_{1}, \phi\right): A \bar{q} \in \operatorname{GMEP}\left(\Theta_{2}, h_{2}, \varphi\right)\right\} \neq \emptyset$.

Remark 1.3. We note that SGMEP in Example 1.1 lies in two different subsets of the same space, while SGMEP in Example 1.2 lies in two different subsets of different spaces.

Let $C$ be a subset of $H$, then a point $x \in C$ is called a fixed point of a nonlinear mapping $T: C \rightarrow C$ if $T x=x$. We denote by $F(T)$, the set of all fixed points of $T$. A nonlinear mapping $T: C \rightarrow C$ is called 
(i) $L$-Lipschitz if there exists $L>0$ such that

$$
\|T x-T y\| \leq L\|x-y\|, \quad \forall x, y \in H ;
$$

(ii) nonexpansive if for all $x, y \in C$, we have

$$
\|T x-T y\| \leq\|x-y\|
$$

(iii) quasi-nonexpansive if $F(T) \neq \emptyset$ and

$$
\|T x-p\| \leq\|x-p\|, \quad \forall x \in C \text { and } p \in F(T) ;
$$

(iv) firmly nonexpansive if for all $x, y \in C$, we have

$$
\|T x-T y\|^{2} \leq\langle T x-T y, x-y\rangle ;
$$

(v) $\beta$-inverse strongly monotone if there exists a constant $\beta>0$, such that

$$
\langle T x-T y, x-y\rangle \geq \beta\|T x-T y\|^{2}, \quad \forall x, y \in H ;
$$

(vi) nonspreading if for all $x, y \in C$, we have

$$
2\|T x-T y\|^{2} \leq\|T x-y\|^{2}+\|x-T y\|^{2} ;
$$

equivalently, $T$ is nonspreading if for all $x, y \in C$,

$$
\|T x-T y\|^{2} \leq\|x-y\|^{2}+2\langle x-T x, y-T y\rangle .
$$

Remark 1.4. We note that every $\beta$-inverse strongly monotone mapping $T$ is $\frac{1}{\beta}$-Lipschitzian, and if $L \in[0,1)$ in (i), then $T$ is called a contraction mapping. It is clear that if $F(T) \neq \emptyset$, then a nonspreading mapping becomes a quasi-nonexpansive mapping. In addition, every nonexpansive mapping with a nonempty set of fixed points is quasi-nonexpansive.

Approximating fixed point solutions of nonexpansive mappings has a variety of applications since many problems can be seen as a fixed point problem of nonexpansive mappings. A significant body of work on iteration methods for fixed point problems has accumulated in the literature (see for example [10-23] and the references therein). Specifically, the Mann algorithm [15], which can be expressed as follows. For each $n \geq 0$,

$$
x_{n+1}=\lambda_{n} x_{n}+\left(1-\lambda_{n}\right) T x_{n},
$$

is often used to approximate a fixed point of a nonexpansive mapping. The iterative sequence $\left\{x_{n}\right\}$ converges weakly to a fixed point of $T$ provided that $\left\{\lambda_{n}\right\} \subset[0,1]$ satisfies

$$
\sum_{n=1}^{\infty} \lambda_{n}\left(1-\lambda_{n}\right)=+\infty
$$

In 2000, Moudafi [24] introduced the viscosity approximation method for approximating fixed points of nonexpansive mappings. Let $f$ be a contraction on $H$, and starting with an arbitrary $x_{0} \in H$, define a sequence $\left\{x_{n}\right\}$ recursively by

$$
x_{n+1}=\lambda_{n} f\left(x_{n}\right)+\left(1-\lambda_{n}\right) T x_{n}, \quad n \geq 0,
$$

where $\left\{\lambda_{n}\right\}$ is a sequence in $(0,1) . \mathrm{Xu}[25]$ proved that if $\left\{\lambda_{n}\right\}$ satisfies certain conditions, the sequence $\left\{x_{n}\right\}$ generated by (6) converges strongly to the unique solution $x \in F(T)$ of the variational inequality

$$
\left\langle(I-f) x^{\dagger}, x-x^{\dagger}\right\rangle \geq 0, \quad \forall x \in F(T) .
$$

Based on the heavy ball methods of the two-order time dynamical system, Polyak [26] first proposed an inertial extrapolation as an acceleration process to solve the smooth convex minimization. The inertial algorithm is a two-step iteration where the next iterate is defined by making use of the previous two iterates. 
Recently, a lot of researchers have constructed some fast iterative algorithms by using inertial extrapolation which includes an inertial proximal method [27, 28], an inertial forward-backward method [29], inertial proximal ADMM [30] and the fast iterative shrinkage thresholding algorithm FISTA [31, 32]. Using the technique of inertial extrapolation, Mainge [33] introduced in 2008 the following inertial Mann algorithm. For each $n \geq 1$, compute

$$
\left\{\begin{array}{l}
y_{n}=x_{n}+\theta_{n}\left(x_{n}-x_{n-1}\right), \\
x_{n+1}=\left(1-\lambda_{n}\right) y_{n}+\lambda_{n} T y_{n} .
\end{array}\right.
$$

Mainge [33] showed that the iterative sequence $\left\{x_{n}\right\}$ converges weakly to a fixed point of $T$ under the following conditions:

(A1) $\theta_{n} \in[0, \alpha)$ for each $n \geq 1$, where $\alpha \in[0,1)$,

(A2) $\sum_{n=1}^{\infty} \theta_{n}\left\|x_{n}-x_{n-1}\right\|^{2}<+\infty$,

(A3) $0<\inf \lambda_{n} \leq \sup \lambda_{n}<1$.

To satisfy the summation condition (A2) of the sequence $\left\{x_{n}\right\}$, one needs to first calculate $\theta_{n}$ at each step (see [28]). In 2015, Bot and Csetnek [34] removed the condition (A2) and substituted (A1) and (A3) with the following conditions:

(B1) for each $n \geq 1,\left\{\theta_{n}\right\} \subset[0, \infty)$ is nondecreasing with $\theta_{1}=0$ and $0 \leq \alpha<1$,

(B2) for each $n \geq 1$,

$$
\delta>\frac{\alpha^{2}(1+\alpha)+\alpha \sigma}{1-\alpha^{2}}, \quad 0 \leq \lambda \leq \lambda_{n} \leq \frac{\delta-\alpha[\alpha(1+\alpha)+\alpha \delta+\sigma]}{\delta[1+\alpha(1+\alpha)+\alpha \delta+\sigma]},
$$

where $\lambda, \sigma, \delta>0$.

By combining the Picard algorithm [35] with the conjugate gradient methods [36], the authors in [37] accelerated the Mann algorithm and obtained the following faster algorithm: For each $n \geq 0$, we compute

$$
\left\{\begin{array}{l}
d_{n+1}=\frac{1}{\lambda}\left(T\left(x_{n}\right)-x_{n}\right)+\beta_{n} d_{n}, \\
y_{n}=x_{n}+\lambda d_{n+1}, \\
x_{n+1}=\mu \alpha_{n} x_{n}+\left(1-\mu \alpha_{n}\right) y_{n},
\end{array}\right.
$$

where $\mu \in(0,1]$ and $\lambda>0$. They proved that the iterative sequence $\left\{x_{n}\right\}$ converges weakly to a fixed point of $T$ provided that the nonnegative sequences $\left\{\alpha_{n}\right\}$ and $\left\{\beta_{n}\right\}$ satisfy the following conditions:

(BB1) $\quad \sum_{n=0}^{\infty} \mu \alpha_{n}\left(1-\mu \alpha_{n}\right)=\infty$,

(BB2) $\quad \sum_{n=0}^{\infty} \beta_{n}<\infty$.

Moreover, the sequence $\left\{x_{n}\right\}$ satisfies the following condition:

$$
\left\{T\left(x_{n}\right)-x_{n}\right\} \text { is bounded. }
$$

Also, they gave some numerical examples to show that the accelerated Mann algorithm is more efficient than the normal Mann algorithm.

In 2016, Suantai et. al. [38] studied the Split Equilibrium Problem which is define as follows: First, we find a point $x^{\star} \in C$ such that

$$
\Theta_{1}\left(x^{\star}, x\right) \geq 0, \forall x \in C \text { and such that } y^{\star}=A x^{\star} \in Q \quad \text { solves } \Theta_{2}\left(y^{\star}, y\right) \geq 0, \forall y \in C,
$$

where $\Theta_{1}: C \times C \rightarrow \mathbb{R}$ and $\Theta_{2}: Q \times Q \rightarrow \mathbb{R}$ are nonlinear bifuncions. The set of solutions of (9) is denoted by $\operatorname{SEP}\left(\Theta_{1}, \Theta_{2}\right)$. The authors in [38] proposed the following iterative algorithm to solve the problem of finding a common element in $\operatorname{SEP}\left(\Theta_{1}, \Theta_{2}\right)$ and a fixed point of a nonspreading multi-valued mapping $S: C \rightarrow K(C)$. Given $x_{1} \in C$, let $\left\{x_{n}\right\}$ be generated by

$$
\left\{\begin{array}{l}
u_{n}=T_{r_{n}}^{\Theta_{1}}\left(I-\gamma A^{\star}\left(I-T_{r_{n}}^{\Theta_{2}}\right) A\right) x_{n}, \\
x_{n+1}=\alpha_{n} x_{n}+\left(1-\alpha_{n}\right) S u_{n}, \quad \forall n \in \mathbb{N},
\end{array}\right.
$$


where $\left\{\alpha_{n}\right\} \subset(0,1), r_{n} \in(0, \infty)$ and $\gamma \in\left(0, \frac{1}{L}\right)$ such that $L$ is the spectral radius of $A^{*} A$, and $A^{\star}$ is the adjoint of $A$. Further, they proved that under certain conditions, the sequence $\left\{x_{n}\right\}$ converges weakly to an element of $F(S) \cap S E P\left(\Theta_{1}, \Theta_{2}\right)$.

More recently, S.H. Rizvi [39] studied the following Split Mixed Equilibrium Problem (SMEP) in real Hilbert spaces. Find a point $x^{*} \in C$ such that

$$
\left\{\begin{array}{l}
\Theta_{1}\left(x^{\star}, x\right)+\left\langle h_{1} x^{\star}, x-x^{\star}\right\rangle \geq 0, \quad \forall x \in C, \\
\text { where } \\
y^{\star}=A x^{\star} \quad \text { solves } \quad \Theta_{2}\left(y^{\star}, y\right)+\left\langle h_{2} y^{\star}, y-y^{\star}\right\rangle \geq 0, \quad \forall y \in Q,
\end{array}\right.
$$

where $h_{1}: C \rightarrow C$ and $h_{2}: Q \rightarrow Q$ are $\theta_{1}, \theta_{2}$-inverse strongly monotone mappings respectively with $\theta=$ $\min \left(\theta_{1}, \theta_{2}\right)$. The set of solutions of (11) is denoted by $\operatorname{SMEP}\left(\Theta_{1}, \Theta_{2}, h_{1}, h_{2}\right)$. Observe that when $\phi=\varphi=0$ in (4), we obtain (11). Thus, Problem (4) is more general than Problem (11). Rizvi [39] introduced the following algorithm for solving (11), as well as fixed point problems for a nonexpansive mapping $S$ in real Hilbert spaces. Let

$$
\left\{\begin{array}{l}
x_{0}=x \in C, \\
y_{n}=T_{r_{n}}^{\Theta_{1}}\left(x_{n}-r_{n} \phi x_{n}\right), \\
v_{n}=T_{r_{n}}^{\Theta_{2}}\left(I-r_{n} \psi\right) A y_{n}, \\
z_{n}=P_{C}\left(y_{n}+\delta A^{\star}\left(v_{n}-A y_{n}\right)\right), \\
x_{n+1}=\beta_{n} x_{n}+\left(1-\beta_{n}\right) S\left[\alpha_{n} u+\left(1-\alpha_{n}\right) z_{n}\right], \quad n \geq 0,
\end{array}\right.
$$

where $P_{C}$ is the metric projection from $H$ onto $C,\left\{r_{n}\right\} \subset(0,2 \theta)$ and $\left\{\alpha_{n}\right\},\left\{\beta_{n}\right\} \subset(0,1)$. Rizvi [39] also proved that under some mild conditions on $\alpha_{n}, \beta_{n}$ and $r_{n}$, the sequence $\left\{x_{n}\right\}$ converges strongly to a solution in $\operatorname{SMEP}\left(\Theta_{1}, \Theta_{2}, h_{1}, h_{2}\right) \cap F(S)$.

Motivated by the above works, it is our aim in this paper to study the SGMEP (4) and introduce a new iterative algorithm for approximating a common solution of (4) and a fixed point problem for nonspreading mappings in real Hilbert spaces. Our algorithm is developed by modifying the accelerated Mann algorithm (8), combined with a modified viscosity approximation method of (6) to obtain a new faster iterative algorithm for finding a common solution of (4) and a fixed point of nonspreading mappings in real Hilbert spaces. Further, our algorithm does not require any prior knowledge of the operator norm. We note here that norms of bounded linear operators are rarely known explicitly (see [40]). Our result is interesting and compliments many recent results previously obtained in this direction in the literature.

\section{Preliminaries}

Let $C$ be a nonempty, closed and convex subset of a real Hilbert space $H$. We denote the strong and weak convergence of a sequence $\left\{x_{n}\right\} \subseteq H$ to a point $p \in H$ by $x_{n} \rightarrow p$ and $x_{n} \rightarrow p$, respectively.

For each point $x \in H$, there exists a unique nearest point in $C$, denoted by $P_{C} x$ such that

$$
\left\|x-P_{C} x\right\| \leq\|x-y\|, \forall y \in C .
$$

The mapping $P_{C}$ is called the metric projection from $H$ onto $C$. It is well known that $P_{C}$ has the following characteristics:

1. $\left\langle x-y, P_{C} x-P_{C} y\right\rangle \geq\left\|P_{C} x-P_{C} y\right\|^{2}$, for every $x, y \in H$;

2. for $x \in H$ and $z \in C, z=P_{C} X \Leftrightarrow$

$$
\langle x-z, z-y\rangle \geq 0, \forall y \in C
$$

3. for $x \in H$ and $y \in C$,

$$
\left\|y-P_{C}(x)\right\|^{2}+\left\|x-P_{C}(x)\right\|^{2} \leq\|x-y\|^{2} .
$$


The following lemmas are useful in establishing our main result.

Lemma 2.1. In a real Hilbert space $H$, the following inequalities hold:

1. $\|x-y\|^{2} \leq\|x\|^{2}-\|y\|^{2}-2\langle x-y, y\rangle$, for all $x, y \in H$;

2. $\|x+y\|^{2} \leq\|x\|^{2}+2\langle y, x+y\rangle$, for all $x, y \in H$;

3. $\|\alpha x+(1-\alpha) y\|^{2}=\alpha\|x\|^{2}+(1-\alpha)\|y\|^{2}-\alpha(1-\alpha)\|x-y\|^{2}$, for all $x, y \in H$ and $\alpha \in[0,1]$.

Lemma 2.2. [41] Let $C$ be a nonempty closed and convex subset of a Hilbert space $H, S: C \rightarrow C$ a nonspreading mapping, and $F(S) \neq \emptyset$. Then $I-S$ is demiclosed at 0 , i.e. for any sequence $\left\{x_{n}\right\} \subset C$ such that $x_{n} \rightarrow z$ and $x_{n}-S x_{n} \rightarrow 0$, then $z \in F(S)$.

For solving the GMEP we make the following assumptions:

Assumption 2.3. Let $C$ be a nonempty closed and convex subset of a real Hilbert space $H$. We make the following assumptions on the bifunction $\Theta: C \times C \rightarrow \mathbb{R}$ :

L1. $\Theta(x, x)=0$, for all $x \in C$;

L2. $\Theta$ is monotone, i.e. $\Theta(x, y)+\Theta(y, x) \leq 0$, for all $x, y \in C$;

L3. for each $x, y, z \in C, \lim _{t \downarrow 0} \Theta(t z+(1-t) x, y) \leq \Theta(x, y)$;

L4. for each $x \in C, y \mapsto \Theta(x, y)$ is convex and lower semicontinuous.

Lemma 2.4. [42] Let $C$ be a nonempty closed and convex subset of a real Hilbert space $H$. Let $\Theta: C \times C \rightarrow \mathbb{R}$ be a bifunction which satisfies Assumption 2.3, $h: C \rightarrow H_{1}$ be a nonlinear mapping, and let $\phi: C \rightarrow \mathbb{R} \cup\{+\infty\}$ be a proper lower semicontinuous and convex function. For $r>0$ and $x \in H_{1}$, define a resolvent function

$$
T_{r}^{\Theta}(x)=\left\{z \in C: \Theta(z, y)+\langle h(z), y-z\rangle+\phi(y)-\phi(z)+\frac{1}{r}\langle y-z, z-x\rangle \geq 0, \quad \forall y \in C\right\},
$$

for all $x \in H$. Then the following conclusions hold:

(i) for each $x \in H, T_{r}^{\Theta}(x) \neq \emptyset$;

(ii) $T_{r}^{\Theta}$ is single-valued;

(iii) $T_{r}^{\Theta}$ is firmly nonexpansive, i.e. for any $x, y \in H$,

$$
\left\|T_{r}^{\Theta} x-T_{r}^{\Theta} y\right\|^{2} \leq\left\langle T_{r}^{\Theta} x-T_{r}^{\Theta} y, x-y\right\rangle
$$

(iv) $F\left(T_{r}^{\Theta}\right)=G M E P\left(\Theta_{1}, h, \phi\right)$;

(v) $\operatorname{GMEP}(\Theta, h, \phi)$ is closed and convex.

Lemma 2.5. [43] Assume $\left\{a_{n}\right\}_{n=1}^{\infty}$ is a sequence of nonnegative real numbers such that

$$
a_{n+1} \leq\left(1-v_{n}\right) a_{n}+v_{n} \delta_{n}, n \geq 0,
$$

where $\left\{v_{n}\right\}_{n=1}^{\infty}$ is a sequence in $(0,1)$ and $\left\{\delta_{n}\right\}_{n=1}^{\infty}$ is a sequence in $\mathbb{R}$ with:

(i) $\sum_{n=0}^{\infty} v_{n}=\infty$;

(ii) $\limsup _{n \rightarrow \infty} \delta_{n} \leq 0$.

Then $\lim _{n \rightarrow \infty} a_{n}=0$.

\section{Main results}

In this section, we introduce a new iterative algorithm with a choice of stepsize which does not depend on the operator norm $\|A\|$. 
Algorithm 3.1. Let $C$ and $Q$ be nonempty closed and convex subsets of real Hilbert spaces $H_{1}$ and $H_{2}$, respectively, and let $A: H_{1} \rightarrow H_{2}$ be a bounded linear operator. Let $\Theta_{1}: C \times C \rightarrow \mathbb{R}$ and $\Theta_{2}: Q \times Q \rightarrow \mathbb{R}$ be bifunctions satisfying Assumption 2.3. Let $h_{1}: C \rightarrow H_{1}$ and $h_{2}: Q \rightarrow H_{2}$ be $\theta_{1}, \theta_{2}$-inverse strongly monotone operators, respectively, such that $\theta=\max \left\{\theta_{1}, \theta_{2}\right\}$. Let $\phi: C \rightarrow \mathbb{R} \cup\{+\infty\}$ and $\varphi: Q \rightarrow \mathbb{R} \cup\{+\infty\}$ be proper, lowersemicontinuous and convex functions, and let $S: C \rightarrow C$ be a nonspreading mapping such that $F(S) \neq \emptyset$. Let $f: H_{1} \rightarrow H_{1}$ be a contraction mapping with constant $\beta \in(0,1)$ and $D$ be a bounded operator with coefficient $\bar{\gamma} \in(0,1)$ such that $0<\xi<\frac{\bar{\gamma}}{\beta}$. Choose an initial point $x_{1} \in H_{1}$ arbitrarily and let $\alpha_{n} \in[0,1]$, $\beta_{n} \in[0,1], w_{n} \in(0,1), r_{n} \in(0,2 \theta)$ and $\lambda>0$. Assume that the $n$th iterate has been constructed, and set $m_{1}=\frac{\gamma_{1} A^{*}\left(T_{r_{1}}^{\theta_{2}}-I\right) A x_{1}}{\lambda}$. We then compute the $(n+1)$ th iterate via the formula

$$
\left\{\begin{array}{l}
m_{n+1}=\frac{\gamma_{n} A^{\star}\left(T_{r_{n}}^{\Theta_{2}}-I\right) A x_{n}}{\lambda}+\beta_{n} m_{n}, \\
y_{n}=x_{n}+\lambda m_{n+1}, \\
z_{n}=T_{r_{n}}^{\Theta_{1}}\left(I-r_{n} h_{1}\right) y_{n}, \\
x_{n+1}=\alpha_{n} \xi f\left(x_{n}\right)+\left(1-\alpha_{n} D\right)\left[\left(1-w_{n}\right) z_{n}+w_{n} S z_{n}\right],
\end{array}\right.
$$

for $n \geq 1$, where $A^{*}$ is the adjoint operator of A. Further, we choose the stepsize $\gamma_{n}$ such that, if

$$
n \in O:=\left\{n:\left(I-T_{r_{n}}^{\Theta_{2}}\right) A x_{n} \neq 0\right\},
$$

then

$$
\gamma_{n} \in\left(0, \frac{2\left\|\left(I-T_{r_{n}}^{\Theta_{2}}\right) x_{n}\right\|^{2}}{\left\|A^{\star}\left(I-T_{r_{n}}^{\Theta_{2}}\right) A x_{n}\right\|^{2}}\right), \quad \forall n \in O
$$

Otherwise, $\gamma_{n}=\gamma(\gamma$ being any nonnegative value).

Remark 3.2. Note that in (14), the choice of stepsize $\gamma_{n}$ is independent of the norm $\|A\|$. The value of $\gamma$ does not influence the considered algorithm but was introduced just for the sake of clarity. Furthermore, we will see from Lemma 3.3 that $\gamma_{n}$ is well defined.

Lemma 3.3. Assume that $\Omega:=\left\{q \in G M E P\left(\Theta_{1}, h_{1}, \phi\right): A q \in G M E P\left(\Theta_{2}, h_{2}, \varphi\right)\right\}$ is nonempty. Then $\gamma_{n}$ defined by (14) is well defined.

Proof. We need to show that $\left\|A^{\star}\left(I-T_{r_{n}}^{\Theta_{2}}\right) A x_{n}\right\|>0$. Take $x \in \Omega$, then $T_{r_{n}}^{\Theta_{1}} x=x$ and $T_{r_{n}}^{\Theta_{2}} A x=A x$, and observe the following:

$$
\begin{aligned}
\left\|\left(I-T_{r_{n}}^{\Theta_{2}}\right) A x_{n}\right\|^{2} & =\left\langle\left(I-T_{r_{n}}^{\Theta_{2}}\right) A x_{n},\left(I-T_{r_{n}}^{\Theta_{2}}\right) A x_{n}\right\rangle \\
& =\left\langle\left(I-T_{r_{n}}^{\Theta_{2}}\right) A x_{n}, A x_{n}-A x+T_{r_{n}}^{\Theta_{2}} A x-T_{r_{n}}^{\Theta_{2}} A x_{n}\right\rangle \\
& =\left\langle\left(I-T_{r_{n}}^{\Theta_{2}}\right) A x_{n}, A x_{n}-A x\right\rangle+\left\langle\left(I-T_{r_{n}}^{\Theta_{2}}\right) A x_{n}, T_{r_{n}}^{\Theta_{2}} A x-T_{r_{n}}^{\Theta_{2}} A x_{n}\right\rangle \\
& =\left\langle A^{\star}\left(I-T_{r_{n}}^{\Theta_{2}}\right) A x_{n}, x_{n}-x\right\rangle+\left\langle\left(I-T_{r_{n}}^{\Theta_{2}}\right) A x_{n}, T_{r_{n}}^{\Theta_{2}} A x-T_{r_{n}}^{\Theta_{2}} A x_{n}\right\rangle \\
& =\left\|A^{*}\left(I-T_{r_{n}}^{\Theta_{2}}\right) A x_{n}\right\| \cdot\left\|x_{n}-x\right\|+\left\|\left(I-T_{r_{n}}^{\Theta_{2}}\right) A x_{n}\right\| \cdot\left\|T_{r_{n}}^{\Theta_{2}} A x-T_{r_{n}}^{\Theta_{2}} A x_{n}\right\| .
\end{aligned}
$$

Consequently, for $n \in O$, that is $\left\|\left(I-T_{r_{n}}^{\Theta_{2}} A x_{n}\right) A x_{n}\right\|>0$, we get $\left\|A^{\star}\left(I-T_{r_{n}}^{\Theta_{2}} A x_{n}\right) A x_{n}\right\| \cdot\left\|x_{n}-x\right\|>0$ and $\left\|\left(I-T_{r_{n}}^{\Theta_{2}}\right) A x_{n}\right\| \cdot\left\|T_{r_{n}}^{\Theta_{2}} A x-T_{r_{n}}^{\Theta_{2}} A x_{n}\right\|>0$. Since $\left\|A^{\star}\left(I-T_{r_{n}}^{\Theta_{2}}\right) A x_{n}\right\| \cdot\left\|x_{n}-x\right\|>0$, we obtain that $\left\|A^{\star}\left(I-T_{r_{n}}^{\Theta_{2}}\right) A x_{n}\right\| \neq$ 0 . This implies that $\gamma_{n}$ is well defined.

We now make the following assumptions:

Assumption 3.4. The sequences $\left\{\alpha_{n}\right\}$ and $\left\{\beta_{n}\right\}$ in Algorithm 3.1 satisfy the following:

$\lim _{n \rightarrow \infty} \alpha_{n}=0$ and $\sum_{n=1}^{\infty} \alpha_{n}=\infty$,

(C3) $\beta_{n} \leq \alpha_{n}^{4}$. 
Furthermore, $\left\{x_{n}\right\}$ satisfies

$$
\left\{\left(T_{r_{n}}^{\Theta_{2}}-I\right) A x_{n}\right\} \text { is bounded. }
$$

Before giving the convergence analysis of Algorithm 3.1, we first show the following result.

Lemma 3.5. Suppose that $\Gamma:=\Omega \cap F(S) \neq \emptyset$ and $\left\{x_{n}\right\}$ is generated by (13). Also, let Assumption 3.4 be satisfied and suppose $r_{n}$ satisfies the following condition:

$$
0<\liminf _{n \rightarrow \infty} r_{n} \leq \limsup _{n \rightarrow \infty} r_{n} \leq 2 \theta .
$$

Then, $\left\{m_{n}\right\}$ and $\left\{x_{n}\right\}$ are bounded, and consequently $\left\{y_{n}\right\}$ is bounded.

Proof. It follows from (C2) that $\lim _{n \rightarrow \infty} \beta_{n}=0$ and so there exists $n_{0} \in \mathbb{N}$ such that $\beta_{n} \leq \frac{1}{2}$ for all $n \geq n_{0}$. Define a number $N_{1}:=\max \left\{\max _{1 \leq k \leq n_{0}}\left\|m_{k}\right\|, \frac{2}{\lambda} \sup _{n \geq 1}\left\|\gamma_{n} A^{\star}\left(T_{r_{n}}^{\Theta_{2}}-I\right) A x_{n}\right\|\right\}$. Then (C4) implies that $N_{1}<\infty$. Assume that $\left\|m_{n}\right\| \leq N_{1}$ for some $n \geq n_{0}$, then the triangle inequality ensures that

$$
\begin{aligned}
\left\|m_{n+1}\right\| & =\left\|\frac{\gamma_{n} A^{\star}\left(T_{r_{n}}^{\Theta_{2}}-I\right) A x_{n}}{\lambda}+\beta_{n} m_{n}\right\| \\
& \leq \frac{1}{\lambda}\left\|\gamma_{n} A^{\star}\left(T_{r_{n}}^{\Theta_{2}}-I\right) A x_{n}\right\|+\beta_{n}\left\|m_{n}\right\| \leq N_{1},
\end{aligned}
$$

which means that $\left\|m_{n+1}\right\| \leq N_{1}$ for all $n \geq 0$, hence $\left\{m_{n}\right\}$ is bounded.

Also, the definition of $\left\{y_{n}\right\}$ implies that

$$
\begin{aligned}
y_{n} & =x_{n}+\lambda\left(\frac{1}{\lambda}\left(\gamma_{n} A^{\star}\left(T_{r_{n}}^{\Theta_{2}}-I\right) A x_{n}\right)+\beta_{n} m_{n}\right) \\
& =x_{n}-\gamma_{n} A^{\star}\left(I-T_{r_{n}}^{\Theta_{2}}\right) A x_{n}+\lambda \beta_{n} m_{n} .
\end{aligned}
$$

Let $p \in \Gamma$, then

$$
\begin{aligned}
\left\|y_{n}-p\right\| & =\left\|x_{n}-\gamma_{n} A^{\star}\left(I-T_{r_{n}}^{\Theta_{2}}\right) A x_{n}+\lambda \beta_{n} m_{n}-p\right\| \\
& \leq\left\|x_{n}-\gamma_{n} A^{\star}\left(I-T_{r_{n}}^{\Theta_{2}}\right) A x_{n}-p\right\|+\lambda \beta_{n}\left\|m_{n}\right\| .
\end{aligned}
$$

Observe that

$$
\begin{aligned}
\left\|x_{n}-\gamma_{n} A^{\star}\left(I-T_{r_{n}}^{\Theta_{2}}\right) A x_{n}-p\right\|^{2}= & \left\|x_{n}-p\right\|^{2}-2 \gamma_{n}\left\langle x_{n}-p, A^{\star}\left(I-T_{r_{n}}^{\Theta_{2}}\right) A x_{n}\right\rangle+\gamma_{n}^{2}\left\|A^{\star}\left(I-T_{r_{n}}^{\Theta_{2}}\right) A x_{n}\right\|^{2} \\
= & \left\|x_{n}-p\right\|^{2}-2 \gamma_{n}\left\langle A x_{n}-A p,\left(I-T_{r_{n}}^{\Theta_{2}}\right) A x_{n}\right\rangle+\gamma_{n}^{2}\left\|A^{\star}\left(I-T_{r_{n}}^{\Theta_{2}}\right) A x_{n}\right\|^{2} \\
= & \left\|x_{n}-p\right\|^{2}-2 \gamma_{n}\left\langle T_{r_{n}}^{\Theta_{2}} A x_{n}-A p,\left(I-T_{r_{n}}^{\Theta_{2}}\right) A x_{n}\right\rangle-2 \gamma_{n}\left\|\left(I-T_{r_{n}}^{\Theta_{2}}\right) A x_{n}\right\|^{2} \\
& +\gamma_{n}^{2}\left\|A^{\star}\left(I-T_{r_{n}}^{\Theta_{2}}\right) A x_{n}\right\|^{2} .
\end{aligned}
$$

Since $T_{r_{n}}^{\Theta_{2}}$ is firmly nonexpansive, then

$$
\left\|T_{r_{n}}^{\Theta_{2}} A x_{n}-A p\right\|^{2} \leq\left\langle T_{r_{n}}^{\Theta_{2}} A x_{n}-A p, A x_{n}-A p\right\rangle,
$$

and so

$$
\left\langle T_{r_{n}}^{\Theta_{2}} A x_{n}-A p, T_{r_{n}}^{\Theta_{2}} A x_{n}-A x_{n}\right\rangle \leq 0
$$

It follows from (17) and (18) that

$$
\begin{aligned}
\left\|x_{n}-\gamma_{n} A^{\star}\left(I-T_{r_{n}}^{\Theta_{2}}\right) A x_{n}-p\right\|^{2} & \leq\left\|x_{n}-p\right\|^{2}-2 \gamma_{n}\left\|\left(I-T_{r_{n}}^{\Theta_{2}}\right) A x_{n}\right\|^{2}+\gamma_{n}^{2}\left\|A^{\star}\left(I-T_{r_{n}}^{\Theta_{2}}\right) A x_{n}\right\|^{2} \\
& =\left\|x_{n}-p\right\|^{2}-\gamma_{n}\left[2\left\|\left(I-T_{r_{n}}^{\Theta_{2}}\right) A x_{n}\right\|^{2}-\gamma_{n}\left\|A^{\star}\left(I-T_{r_{n}}^{\Theta_{2}}\right) A x_{n}\right\|^{2}\right] \\
& \leq\left\|x_{n}-p\right\|^{2} .
\end{aligned}
$$

Therefore, from (16) and (19), we get

$$
\left\|y_{n}-p\right\| \leq\left\|x_{n}-p\right\|+\lambda \beta_{n} N_{1} .
$$


Again from (13), we use the fact that $T_{r_{n}}^{\Theta_{1}}$ is firmly nonexpansive to show that

$$
\begin{aligned}
\left\|z_{n}-p\right\|^{2} & =\left\|T_{r_{n}}^{\Theta_{1}}\left(I-r_{n} h_{1}\right) y_{n}-T_{r_{n}}^{\Theta_{1}}\left(I-r_{n} h_{1}\right) p\right\|^{2} \\
& \leq\left\|\left(I-r_{n} h_{1}\right) y_{n}-\left(I-r_{n} h_{1}\right) p\right\|^{2} \\
& =\left\|\left(y_{n}-p\right)-r_{n}\left(h_{1} y_{n}-h_{1} p\right)\right\|^{2} \\
& =\left\|y_{n}-p\right\|^{2}-2 r_{n}\left\langle y_{n}-p, h_{1} y_{n}-h_{1} p\right\rangle+r_{n}^{2}\left\|h_{1} y_{n}-h_{1} p\right\|^{2} \\
& \leq\left\|y_{n}-p\right\|^{2}-2 r_{n} \theta\left\|h_{1} y_{n}-h_{1} p\right\|^{2}+r_{n}^{2}\left\|h_{1} y_{n}-h_{1} p\right\|^{2} \\
& =\left\|y_{n}-p\right\|^{2}-r_{n}\left(2 \theta-r_{n}\right)\left\|h_{1} y_{n}-h_{1} p\right\|^{2} .
\end{aligned}
$$

By condition (C5), we obtain

$$
\left\|z_{n}-p\right\|^{2} \leq\left\|y_{n}-p\right\|^{2} .
$$

Now define $U_{n}=\left(1-w_{n}\right) I+w_{n} S$, and observe that

$$
\begin{aligned}
\left\|U_{n} z_{n}-p\right\| & =\left\|\left(1-w_{n}\right)\left(z_{n}-p\right)+w_{n}\left(S z_{n}-p\right)\right\| \\
& \leq\left(1-w_{n}\right)\left\|z_{n}-p\right\|+w_{n}\left\|S z_{n}-p\right\| \\
& \leq\left(1-w_{n}\right)\left\|z_{n}-p\right\|+w_{n}\left\|z_{n}-p\right\| \\
& =\left\|z_{n}-p\right\| .
\end{aligned}
$$

Therefore, from (13), (20) and (22), we have

$$
\begin{aligned}
\left\|x_{n+1}-p\right\| & =\left\|\alpha_{n}\left(\xi f\left(x_{n}\right)-D p\right)+\left(1-\alpha_{n} D\right)\left(U_{n} z_{n}-p\right)\right\| \\
& \leq \alpha_{n}\left\|\xi f\left(x_{n}\right)-D p\right\|+\left(1-\alpha_{n} \bar{\gamma}\right)\left\|U_{n} z_{n}-p\right\| \\
& \leq \alpha_{n}\left[\left\|\xi\left(f\left(x_{n}\right)-f(p)\right)+(\xi f(p)-D p)\right\|\right]+\left(1-\alpha_{n} \bar{\gamma}\right)\left\|z_{n}-p\right\| \\
& \leq \alpha_{n} \xi \beta\left\|x_{n}-p\right\|+\alpha_{n}\|\xi f(p)-D p\|+(1-\alpha \bar{\gamma})\left[\left\|x_{n}-p\right\|+\lambda \beta_{n} N_{1}\right] \\
& =\left(1-\alpha_{n}(\bar{\gamma}-\xi \beta)\right)\left\|x_{n}-p\right\|+\alpha_{n}\|\xi f(p)-D p\|+\lambda \beta_{n} N_{1} \\
& \leq \max \left\{\left\|x_{n}-p\right\|, \frac{\|\xi f(p)-D p\|}{\bar{\gamma}-\xi \beta}+\frac{\lambda N_{1}}{\bar{\gamma}-\xi \beta}\right\} \\
& \vdots \\
& \leq \max \left\{\left\|x_{1}-p\right\|, \frac{\|\xi f(p)-D p\|}{\bar{\gamma}-\xi \beta}+\frac{\lambda N_{1}}{\bar{\gamma}-\xi \beta}\right\} .
\end{aligned}
$$

This implies that $\left\{x_{n}\right\}$ is bounded. It follows from (20) that $\left\{y_{n}\right\}$ is also bounded.

Theorem 3.6. Let $C$ and $Q$ be nonempty closed and convex subsets of real Hilbert spaces $H_{1}$ and $H_{2}$, respectively, and $A: H_{1} \rightarrow H_{2}$ a bounded linear operator. Let $\Theta_{1}: C \times C \rightarrow \mathbb{R}$ and $\Theta_{2}: Q \times Q \rightarrow \mathbb{R}$ be bifunctions satisfying Assumption 2.3. Let $h_{1}: C \rightarrow H_{1}$ and $h_{2}: Q \rightarrow H_{2}$ be $\theta_{1}, \theta_{2}$-inverse strongly monotone mappings, respectively, such that $\theta=\max \left\{\theta_{1}, \theta_{2}\right\}$. Let $\phi: C \rightarrow \mathbb{R} \cup\{+\infty\}$ and $\varphi: Q \rightarrow \mathbb{R} \cup\{+\infty\}$ be proper, lowersemicontinuous and convex functions, and let $S: C \rightarrow C$ be a nonspreading mapping such that $F(S) \neq \emptyset$. Let $f: H_{1} \rightarrow H_{1}$ be a contraction mapping with constant $\beta \in(0,1)$, and let $D$ a bounded operator with coefficient $\bar{\gamma} \in(0,1)$ such that $0<\xi<\frac{\bar{\gamma}}{\beta}$. Choose an initial value $x_{1} \in H_{1}$ arbitrarily and let $\alpha_{n} \in[0,1], \beta_{n} \in[0,1]$, $w_{n} \in(0,1), r_{n} \in(0,2 \theta)$ and $\lambda>0$. Suppose $\Gamma:=\Omega \cap F(S) \neq \emptyset$, Assumption 3.4, condition (C5) and the following are satisfied:

(C6) $\quad \liminf _{n \rightarrow \infty} r_{n}>0$;

(C7) $0<\liminf _{n \rightarrow \infty} w_{n} \leq \lim \sup _{n \rightarrow \infty} w_{n}<1$.

Then the sequences $\left\{x_{n}\right\},\left\{y_{n}\right\}$ and $\left\{z_{n}\right\}$ generated by Algorithm 3.1 converge strongly to a point $z$, where $z=$ $P_{\Gamma}(I-D+\xi f)(z)$ is a unique solution of the variational inequality

$$
\langle(D-\xi f) z, z-x\rangle \leq 0, \quad x \in \Gamma .
$$


Proof. Let $p \in \Gamma$, then from Lemma 2.1(2) and (19), we have

$$
\begin{aligned}
\left\|y_{n}-p\right\|^{2} & =\left\|x_{n}-\gamma_{n} A^{\star}\left(I-T_{r_{n}}^{\Theta_{2}}\right) A x_{n}-p+\lambda \beta_{n} m_{n}\right\|^{2} \\
& \leq\left\|x_{n}-\gamma_{n} A^{\star}\left(I-T_{r_{n}}^{\Theta_{2}}\right) A x_{n}-p\right\|^{2}+2 \lambda \beta_{n}\left\langle y_{n}-p, m_{n}\right\rangle \\
& \leq\left\|x_{n}-p\right\|^{2}+\beta_{n} \rho_{n},
\end{aligned}
$$

where $\rho_{n}:=\left\{2 \lambda\left\langle y_{n}-p, m_{n}\right\rangle\right\}$. Using Lemma 3.5, it follows that $\left\{\rho_{n}\right\}$ is bounded. Thus, there exists $N_{2}>0$ such that $\rho_{n} \leq N_{2}$ for all $n \geq 1$. Hence, it follows from condition (C3) that

$$
\left\|y_{n}-p\right\|^{2} \leq\left\|x_{n}-p\right\|^{2}+2 \alpha_{n}^{4} N_{2} \text {. }
$$

Furthermore, from (22) and (26), we have

$$
\begin{aligned}
\left\|x_{n+1}-p\right\|^{2}= & \left\|\alpha_{n}\left(\xi f\left(x_{n}\right)-D p\right)+\left(1-\alpha_{n} D\right)\left(U_{n} z_{n}-p\right)\right\|^{2} \\
\leq & \left\|\left(1-\alpha_{n} D\right)\left(U_{n} z_{n}-p\right)\right\|^{2}+2 \alpha_{n}\left\langle\xi f\left(x_{n}\right)-D p, x_{n+1}-p\right\rangle \\
\leq & \left(1-\alpha_{n} \bar{\gamma}\right)^{2}\left\|z_{n}-p\right\|^{2}+2 \alpha_{n} \xi\left\langle f\left(x_{n}\right)-f(p), x_{n+1}-p\right\rangle+2 \alpha_{n}\left\langle\xi f(p)-D p, x_{n+1}-p\right\rangle \\
\leq & \left(1-\alpha_{n} \bar{\gamma}\right)^{2}\left\|y_{n}-p\right\|^{2}+2 \alpha_{n} \xi\left\langle f\left(x_{n}\right)-f(p), x_{n+1}-p\right\rangle+2 \alpha_{n}\left\langle\xi f(p)-D p, x_{n+1}-p\right\rangle \\
\leq & \left(1-\alpha_{n} \bar{\gamma}\right)^{2}\left[\left\|x_{n}-p\right\|^{2}+2 \alpha^{4} N_{2}\right]+2 \alpha_{n} \xi \beta\left\|x_{n}-p\right\| \cdot\left\|x_{n+1}-p\right\| \\
& +2 \alpha_{n}\left\langle\xi f(p)-D p, x_{n+1}-p\right\rangle .
\end{aligned}
$$

We now divide the remaining proof of the theorem into two cases.

Case I: Suppose there exists $n_{0} \in \mathbb{N}$ such that $\left\{\left\|x_{n}-p\right\|\right\}$ is monotonically decreasing for all $n \geq n_{0}$. Then $\left\{\left\|x_{n}-p\right\|\right\}$ converges as $n \rightarrow \infty$ and so

$$
\left\|x_{n}-p\right\|^{2}-\left\|x_{n+1}-p\right\|^{2} \rightarrow 0, \quad n \rightarrow \infty .
$$

Note that, from (19), (25) and (26), we obtain

$$
\left\|y_{n}-p\right\|^{2} \leq\left\|x_{n}-p\right\|^{2}-\gamma_{n}\left[2\left\|\left(I-T_{r_{n}}^{\Theta_{2}}\right) A x_{n}\right\|^{2}-\gamma_{n}\left\|A^{\star}\left(I-T_{r_{n}}^{\Theta_{2}}\right) A x_{n}\right\|^{2}\right]+2 \alpha_{n}^{4} N_{2} .
$$

Also from (27), we obtain

$$
\begin{aligned}
\left\|x_{n+1}-p\right\|^{2} & \leq\left(1-\alpha_{n} \bar{\gamma}\right)^{2} \mid\left\|y_{n}-p\right\|^{2}+2 \alpha_{n} \xi\left\langle f\left(x_{n}\right)-f(p), x_{n+1}-p\right\rangle+2 \alpha_{n}\left\langle\xi f(p)-D p, x_{n+1}-p\right\rangle \\
& \leq\left\|y_{n}-p\right\|^{2}+2 \alpha_{n} \xi\left\langle f\left(x_{n}\right)-f(p), x_{n+1}-p\right\rangle+2 \alpha_{n}\left\langle\xi f(p)-D p, x_{n+1}-p\right\rangle .
\end{aligned}
$$

Substituting (28) into (29), we have

$$
\begin{aligned}
\left\|x_{n+1}-p\right\|^{2} \leq & \left\|x_{n}-p\right\|^{2}-\gamma_{n}\left[2\left\|\left(I-T_{r_{n}}^{\Theta_{2}}\right) A x_{n}\right\|^{2}-\gamma_{n}\left\|A^{\star}\left(I-T_{r_{n}}^{\Theta_{2}}\right) A x_{n}\right\|^{2}\right] \\
& +2 \alpha_{n} \xi\left\langle f\left(x_{n}\right)-f(p), x_{n+1}-p\right\rangle+2 \alpha_{n}\left\langle\xi f(p)-D p, x_{n+1}-p\right\rangle+2 \alpha_{n}^{4} N_{2} .
\end{aligned}
$$

Putting $\Lambda_{n}:=2\left\|I-T_{r_{n}}^{\Theta_{2}} A x_{n}\right\|^{2}-\gamma_{n}\left\|A^{\star}\left(I-T_{r_{n}}^{\Theta_{2}}\right) A x_{n}\right\|^{2}$, then since $\alpha_{n} \rightarrow 0$, as $n \rightarrow \infty$, it follows from (30) that

$$
\begin{aligned}
\gamma_{n} \Lambda_{n} \leq & \left\|x_{n}-p\right\|^{2}-\left\|x_{n+1}-p\right\|^{2}+2 \alpha_{n} \xi\left\langle f\left(x_{n}\right)-f(p), x_{n+1}-p\right\rangle \\
& +2 \alpha_{n}\left\langle\xi f(p)-D p, x_{n+1}-p\right\rangle+2 \alpha_{n}^{4} N_{2} \rightarrow 0 .
\end{aligned}
$$

From the condition on the stepsize given by (14), for a small $\epsilon>0$, we know that

$$
\gamma_{n}<\frac{2\left\|\left(I-T_{r_{n}}^{\Theta_{2}}\right) A x_{n}\right\|^{2}}{\left\|A^{\star}\left(I-T_{r_{n}}^{\Theta_{2}}\right) A x_{n}\right\|^{2}}-\epsilon,
$$

which implies

$$
\gamma_{n}\left\|A^{\star}\left(I-T_{r_{n}}^{\Theta_{2}}\right) A x_{n}\right\|^{2}<2\left\|\left(I-T_{r_{n}}^{\Theta_{2}}\right) A x_{n}\right\|^{2}-\epsilon\left\|A^{\star}\left(I-T_{r_{n}}^{\Theta_{2}}\right) A x_{n}\right\|^{2}
$$

and thus we have

$$
\epsilon\left\|A^{\star}\left(I-T_{r_{n}}^{\Theta_{2}}\right) A x_{n}\right\|^{2}<2\left\|\left(I-T_{r_{n}}^{\Theta_{2}}\right) A x_{n}\right\|^{2}-\gamma_{n}\left\|A^{\star}\left(I-T_{r_{n}}^{\Theta_{2}}\right) A x_{n}\right\|^{2} .
$$


This implies that

$$
\epsilon\left\|A^{\star}\left(I-T_{r_{n}}^{\Theta_{2}}\right) A x_{n}\right\|^{2}<\Lambda_{n} \rightarrow 0 \text {, as } n \rightarrow \infty .
$$

Hence

$$
\lim _{n \rightarrow \infty}\left\|A^{\star}\left(I-T_{r_{n}}^{\Theta_{2}}\right) A x_{n}\right\|^{2}=0 .
$$

Further, from (31) and (33), we get

$$
\begin{aligned}
0<\epsilon \|(I- & \left.T_{r_{n}}^{\Theta_{2}}\right) A x_{n}\left\|^{2} \leq \gamma_{n}\right\|\left(I-T_{r_{n}}^{\Theta_{2}}\right) A x_{n} \|^{2} \\
& \leq\left\|x_{n}-p\right\|^{2}-\left\|x_{n+1}-p\right\|^{2}+\gamma_{n}^{2}\left\|A^{\star}\left(I-T_{r_{n}}^{\Theta_{2}}\right) A x_{n}\right\|^{2}+2 \alpha_{n} \xi\left\langle f\left(x_{n}\right)-f(p), x_{n+1}-p\right\rangle \\
& +2 \alpha_{n}\left\langle\xi f(p)-D p, x_{n+1}-p\right\rangle+2 \alpha_{n}^{4} N_{2} \rightarrow 0, \text { as } n \rightarrow \infty,
\end{aligned}
$$

and hence

$$
\lim _{n \rightarrow \infty}\left\|\left(I-T_{r_{n}}^{\Theta_{2}}\right) A x_{n}\right\|=0
$$

Also from (27), we obtain

$$
\begin{aligned}
\left\|x_{n+1}-p\right\|^{2} & \leq\left(1-\alpha_{n} \bar{\gamma}\right)^{2}\left\|z_{n}-p\right\|^{2}+2 \alpha_{n} \xi\left\langle f\left(x_{n}\right)-f(p), x_{n+1}-p\right\rangle+2 \alpha_{n}\left\langle\xi f(p)-D p, x_{n+1}-p\right\rangle \\
& \leq\left\|z_{n}-p\right\|^{2}+2 \alpha_{n} \xi \beta\left\|x_{n}-p\right\| \cdot\left\|x_{n+1}-p\right\|+2 \alpha_{n}\left\langle\xi f(p)-D p, x_{n+1}-p\right\rangle .
\end{aligned}
$$

Substituting (21) into (36), and from (26), we have

$$
\begin{aligned}
\left\|x_{n+1}-p\right\|^{2} \leq & \left\|y_{n}-p\right\|^{2}-r_{n}\left(2 \theta-r_{n}\right)\left\|h_{1} y_{n}-h_{1} p\right\|^{2}+2 \alpha_{n} \xi \beta\left\|x_{n}-p\right\| \cdot\left\|x_{n+1}-p\right\| \\
& +2 \alpha_{n}\left\langle\xi f(p)-D p, x_{n+1}-p\right\rangle \\
\leq & \left\|x_{n}-p\right\|^{2}+2 \alpha_{n}^{4} N_{2}-r_{n}\left(2 \theta-r_{n}\right)\left\|h_{1} y_{n}-h_{1} p\right\|^{2}+2 \alpha_{n} \xi \beta\left\|x_{n}-p\right\| \cdot\left\|x_{n+1}-p\right\| \\
& +2 \alpha_{n}\left\langle\xi f(p)-D p, x_{n+1}-p\right\rangle .
\end{aligned}
$$

Thus, we have

$$
\begin{aligned}
r_{n}\left(2 \theta-r_{n}\right)\left\|h_{1} y_{n}-h_{1} p\right\|^{2} \leq & \left\|x_{n}-p\right\|^{2}-\left\|x_{n+1}-p\right\|^{2}+2 \alpha_{n} \xi \beta\left\|x_{n}-p\right\| \cdot\left\|x_{n+1}-p\right\| \\
& +2 \alpha_{n}\left\langle\xi f(p)-D p, x_{n+1}-p\right\rangle \rightarrow 0, \text { as } n \rightarrow \infty .
\end{aligned}
$$

Since $\left\{r_{n}\right\} \subset(0,2 \theta)$, we conclude that

$$
\lim _{n \rightarrow \infty}\left\|h_{1} y_{n}-h_{1} p\right\|^{2}=0 .
$$

Further, observe that

$$
\begin{aligned}
\left\|z_{n}-p\right\|^{2} & =\left\|T_{r_{n}}^{\Theta_{1}}\left(y_{n}-r_{n} h_{1} y_{n}\right)-T_{r_{n}}^{\Theta_{1}}\left(p-r_{n} h_{1} p\right)\right\|^{2} \\
& \leq\left\langle z_{n}-p,\left(y_{n}-r_{n} h_{1} y_{n}\right)-\left(p-r_{n} h_{1} p\right)\right\rangle \\
& \leq \frac{1}{2}\left\{\left\|z_{n}-p\right\|^{2}+\left\|\left(y_{n}-r_{n} h_{1} y_{n}\right)-\left(p-r_{n} h_{1} p\right)\right\|^{2}-\left\|\left(z_{n}-p\right)-\left[\left(y_{n}-r_{n} h_{1} y_{n}\right)-\left(p-r_{n} h_{1} p\right)\right]\right\|^{2}\right\} .
\end{aligned}
$$

Hence

$$
\begin{aligned}
\left\|z_{n}-p\right\|^{2} & \leq\left\|\left(y_{n}-r_{n} h_{1} y_{n}\right)-\left(p-r_{n} h_{1} p\right)\right\|^{2}-\left\|\left(z_{n}-y_{n}\right)+r_{n}\left(h_{1} y_{n}-h_{1} p\right)\right\|^{2} \\
& \leq\left\|y_{n}-p\right\|^{2}-\left\|z_{n}-y_{n}\right\|^{2}+2 r_{n}\left\|z_{n}-y_{n}\right\| \cdot\left\|h_{1} y_{n}-h_{1} p\right\|^{2} .
\end{aligned}
$$

From (36) and (39), we obtain

$$
\begin{aligned}
\left\|x_{n+1}-p\right\|^{2} \leq & \left\|y_{n}-p\right\|^{2}-\left\|z_{n}-y_{n}\right\|^{2}+2 r_{n}\left\|z_{n}-y_{n}\right\| \cdot\left\|h_{1} y_{n}-h_{1} p\right\|^{2}+2 \alpha_{n} \xi \beta\left\|x_{n}-p\right\| \cdot\left\|x_{n+1}-p\right\| \\
& +2 \alpha_{n}\left\langle\xi f(p)-D p, x_{n+1}-p\right\rangle \\
\leq & \left\|x_{n}-p\right\|^{2}+2 \alpha_{n}^{4} N_{2}-\left\|z_{n}-y_{n}\right\|^{2}+2 r_{n}\left\|z_{n}-y_{n}\right\| \cdot \mid h_{1} y_{n}-h_{1} p \|^{2} \\
& +2 \alpha_{n} \xi \beta\left\|x_{n}-p\right\| \cdot\left\|x_{n+1}-p\right\|+2 \alpha_{n}\left\langle\xi f(p)-D p, x_{n+1}-p\right\rangle .
\end{aligned}
$$


Therefore

$$
\begin{aligned}
\left\|z_{n}-y_{n}\right\|^{2} \leq & \left\|x_{n}-p\right\|^{2}-\left\|x_{n+1}-p\right\|^{2}+2 \alpha_{n}^{4} N_{2}+2 r_{n}\left\|z_{n}-y_{n}\right\| \cdot\left\|h_{1} y_{n}-h_{1} p\right\|^{2} \\
& +2 \alpha_{n} \xi \beta\left\|x_{n}-p\right\| \cdot\left\|x_{n+1}-p\right\|+2 \alpha_{n}\left\langle\xi f(p)-D p, x_{n+1}-p\right\rangle .
\end{aligned}
$$

Since $\alpha_{n} \rightarrow 0$ as $n \rightarrow \infty$, and using (38), we obtain

$$
\lim _{n \rightarrow \infty}\left\|z_{n}-y_{n}\right\|^{2}=0
$$

Moreover

$$
\begin{aligned}
\left\|U_{n} z_{n}-p\right\|^{2} & =\left\|\left(1-w_{n}\right) z_{n}+w_{n} S z_{n}-p\right\|^{2} \\
& \leq\left(1-w_{n}\right)\left\|z_{n}-p\right\|^{2}+w_{n}\left\|S z_{n}-p\right\|^{2}-w_{n}\left(1-w_{n}\right)\left\|S z_{n}-z_{n}\right\|^{2} \\
& \leq\left(1-w_{n}\right)\left\|z_{n}-p\right\|^{2}+w_{n}\left\|z_{n}-p\right\|^{2}-w_{n}\left(1-w_{n}\right)\left\|S z_{n}-z_{n}\right\|^{2} \\
& =\left\|z_{n}-p\right\|^{2}-w_{n}\left(1-w_{n}\right)\left\|S z_{n}-z_{n}\right\|^{2} \\
& \leq\left\|x_{n}-p\right\|^{2}+2 \alpha_{n}^{4} N_{2}-w_{n}\left(1-w_{n}\right)\left\|S z_{n}-z_{n}\right\|^{2} .
\end{aligned}
$$

Note that from (27), we have

$$
\begin{aligned}
\left\|x_{n+1}-p\right\|^{2} & \leq\left(1-\alpha_{n} \bar{\gamma}\right)^{2}\left\|U_{n} z_{n}-p\right\|^{2}+2 \alpha_{n}\left\langle\xi f\left(x_{n}\right)-D p, x_{n+1}-p\right\rangle \\
& \leq\left\|U_{n} z_{n}-p\right\|^{2}+2 \alpha_{n}\left\langle\xi f\left(x_{n}\right)-D p, x_{n+1}-p\right\rangle,
\end{aligned}
$$

then from (41) and (42), we get

$$
\begin{aligned}
w_{n}\left(1-w_{n}\right)\left\|S z_{n}-z_{n}\right\|^{2} \leq & \left\|x_{n}-p\right\|^{2}-\left\|x_{n+1}-p\right\|^{2}+2 \alpha_{n}\left\langle\xi f\left(x_{n}\right)-D p, x_{n+1}-p\right\rangle \\
& +2 \alpha_{n}^{4} N_{2} \rightarrow 0, \text { as } n \rightarrow \infty .
\end{aligned}
$$

By condition (C7), we have

$$
\lim _{n \rightarrow \infty}\left\|S z_{n}-z_{n}\right\|=0
$$

Also

$$
\left\|U_{n} z_{n}-z_{n}\right\|=w_{n}\left\|S z_{n}-z_{n}\right\| \rightarrow 0, \text { as } n \rightarrow \infty \text {. }
$$

It is clear from (3.1) that

$$
\left\|x_{n+1}-U_{n} z_{n}\right\|=\alpha_{n}\left\|\xi f\left(x_{n}\right)-D U_{n} z_{n}\right\| \rightarrow 0, \text { as } n \rightarrow \infty \text {, }
$$

and

$$
\left\|y_{n}-x_{n}\right\| \rightarrow 0, \quad n \rightarrow \infty
$$

then, it follows from (40) and (46) that

$$
\left\|z_{n}-x_{n}\right\| \leq\left\|z_{n}-y_{n}\right\|+\left\|y_{n}-x_{n}\right\| \rightarrow 0 \text {, as } n \rightarrow \infty \text {. }
$$

Furthermore, it follows from (44), (45) and (47) that

$$
\left\|x_{n+1}-x_{n}\right\| \leq\left\|x_{n+1}-U_{n} z_{n}\right\|+\left\|U_{n} z_{n}-z_{n}\right\|+\left\|z_{n}-x_{n}\right\| \rightarrow 0 \text {, as } n \rightarrow \infty \text {. }
$$

Since $\left\{x_{n}\right\}$ is bounded, there exists a subsequence $\left\{x_{n_{j}}\right\}$ of $\left\{x_{n}\right\}$ such that $x_{n_{j}} \rightarrow \bar{x}$. It follows from (46) and (47) that $y_{n_{j}} \rightarrow \bar{x}$ and $z_{n_{j}} \rightarrow \bar{x}$, respectively. Since $\lim _{n \rightarrow \infty}\left\|S z_{n}-z_{n}\right\|=0$, and by Lemma 2.2, we have $\bar{x} \in F(S)$. Next, we show that $\bar{x} \in \Omega$. Since $z_{n}=T_{r_{n}}^{\Theta_{1}}\left(y_{n}-r_{n} h_{1} y_{n}\right)$, then

$$
\Theta_{1}\left(z_{n}, y\right)+\left\langle h_{1} z_{n}, y-z_{n}\right\rangle+\phi(y)-\phi\left(z_{n}\right)+\frac{1}{r_{n}}\left\langle y-z_{n}, z_{n}-y_{n}\right\rangle \geq 0, \quad \forall y \in C
$$


It follows from the monotonicity of $\Theta_{1}$ that

$$
\left\langle h_{1} z_{n}, y-z_{n}\right\rangle+\phi(y)-\phi\left(z_{n}\right)+\frac{1}{r_{n}}\left\langle y-z_{n}, z_{n}-y_{n}\right\rangle \geq \Theta_{1}\left(y, z_{n}\right) .
$$

Replacing $n$ by $n_{j}$, we get

$$
\left\langle h_{1} z_{n_{j}}, y-z_{n_{j}}\right\rangle+\frac{1}{r_{n_{j}}}\left\langle y-z_{n_{j}}, z_{n_{j}}-y_{n_{j}}\right\rangle \geq \Theta_{1}\left(y, z_{n_{j}}\right)+\phi\left(z_{n_{j}}\right)-\phi(y) .
$$

Further, for any $t \in(0,1]$ and $y \in C$, let $y_{t}=t y+(1-t) \bar{x}$. Since $\bar{x} \in C$ and $y \in C$, then $y_{t} \in C$. So from (48), we have

$$
\begin{aligned}
\left\langle y_{t}-z_{n_{j}}, h_{1} y_{t}\right\rangle \geq & \left\langle y_{t}-z_{n_{j}}, h_{1} y_{t}\right\rangle-\left\langle y_{t}-z_{n_{j}}, h_{1} y_{n_{j}}\right\rangle-\left\langle y_{t}-z_{n_{j}}, \frac{z_{n_{j}}-y_{n_{j}}}{r_{n_{j}}}\right\rangle+\Theta_{1}\left(y_{t}, z_{n_{j}}\right) \\
& +\phi\left(z_{n_{j}}\right)-\phi\left(y_{t}\right) \\
= & \left\langle y_{t}-z_{n_{j}}, h_{1} y_{t}-h_{1} z_{n_{j}}\right\rangle+\left\langle y_{t}-z_{n_{j}}, h_{1} z_{n_{j}}-h_{1} y_{n_{j}}\right\rangle-\left\langle y_{t}-z_{n_{j}}, \frac{z_{n_{j}}-y_{n_{j}}}{r_{n_{j}}}\right\rangle+\Theta_{1}\left(y_{t}, z_{n_{j}}\right) \\
& +\phi\left(z_{n_{j}}\right)-\phi\left(y_{t}\right) .
\end{aligned}
$$

From the Lipschitz continuity of $h_{1}$ and $\lim _{n \rightarrow \infty}|| z_{n}-y_{n} \|=0$, we obtain $\left\|h_{1} z_{n_{j}}-h_{1} y_{n_{j}}\right\| \rightarrow 0$, as $n \rightarrow \infty$. Also since $h_{1}$ is monotone, we have $\left\langle y_{t}-z_{n_{j}}, h_{1} y_{t}-h_{1} z_{n_{j}}\right\rangle \geq 0$. Therefore, by L4 and the weak lower semicontinuity of $\phi$, taking the limit of (49) as $j \rightarrow \infty$, we have

$$
\left\langle y_{t}-\bar{x}, h_{1} y_{t}\right\rangle \geq \Theta_{1}\left(y_{t}, \bar{x}\right)+\phi(\bar{x})-\phi\left(y_{t}\right) .
$$

Hence, from L1 and (50), we get

$$
\begin{aligned}
0 & =\Theta_{1}\left(y_{t}, y_{t}\right)+\phi\left(y_{t}\right)-\phi\left(y_{t}\right) \\
& \leq t \Theta_{1}\left(y_{t}, y\right)+(1-t) \Theta_{1}\left(y_{t}, \bar{x}\right)+t \phi(y)+(1-t) \phi(\bar{x})-\phi\left(y_{t}\right) \\
& =t\left(\Theta_{1}\left(y_{t}, y\right)+\phi(y)-\phi\left(y_{t}\right)\right)+(1-t)\left(\Theta_{1}\left(y_{t}, \bar{x}\right)+\phi(\bar{x})-\phi\left(y_{t}\right)\right) \\
& \leq t\left(\Theta_{1}\left(y_{t}, y\right)+\phi(y)-\phi\left(y_{t}\right)\right)+(1-t)\left\langle y_{t}-\bar{x}, h_{1} y_{t}\right\rangle \\
& \leq t\left(\Theta_{1}\left(y_{t}, y\right)+\phi(y)-\phi\left(y_{t}\right)\right)+(1-t) t\left\langle y-\bar{x}, h_{1} y_{t}\right\rangle,
\end{aligned}
$$

which implies that

$$
\Theta_{1}\left(y_{t}, y\right)+(1-t)\left\langle y-\bar{x}, h_{1} y_{t}\right\rangle+\phi(y)-\phi\left(y_{t}\right) \geq 0
$$

Letting $t \rightarrow 0$, we have

$$
\Theta_{1}(\bar{x}, y)+\left\langle y-\bar{x}, h_{1} \bar{x}\right\rangle+\phi(y)-\phi(\bar{x}) \geq 0, \quad y \in C,
$$

which implies that $\bar{x} \in \operatorname{GMEP}\left(\Theta_{1}, h, \phi\right)$.

Since $A$ is a bounded linear operator, $A x_{n_{j}} \rightarrow A \bar{x}$. It follows from (35) that

$$
T_{r_{n_{j}}}^{\Theta_{2}} A x_{n_{j}} \rightarrow A \bar{x}, \text { as } j \rightarrow \infty .
$$

By the definition of $T_{r_{n_{j}}}^{\Theta_{2}} A x_{n_{j}}$, we have

$$
\begin{aligned}
& \Theta_{2}\left(T_{r_{n_{j}}}^{\Theta_{2}} A x_{n_{j}}, g\right)+\left\langle h_{2}\left(T_{r_{n_{j}}}^{\Theta_{2}} A x_{n_{j}}\right), g-T_{r_{n_{j}}}^{\Theta_{2}} A x_{n_{j}}\right\rangle+\varphi(g)-\varphi\left(T_{r_{n_{j}}}^{\Theta_{2}} A x_{n_{j}}\right) \\
& +\frac{1}{r_{n_{j}}}\left\langle y-T_{r_{n_{j}}}^{\Theta_{2}} A x_{n_{j}}, T_{r_{n_{j}}}^{\Theta_{2}} A x_{n_{j}}-A x_{n_{j}}\right\rangle \geq 0, \quad \forall g \in Q \quad \text { and } y \in H_{2} .
\end{aligned}
$$

Since $\Theta_{2}$ is upper semicontinuous in the first argument, taking limsup of the above inequality as $j \rightarrow \infty$, we get

$$
\Theta_{2}(A \bar{x}, g)+\left\langle h_{2}(A \bar{x}), g-A \bar{x}\right\rangle+\varphi(g)-\varphi(A \bar{x}) \geq 0, \quad \forall g \in Q,
$$

which implies $A \bar{x} \in \operatorname{GMEP}\left(\Theta_{2}, h_{2}, \varphi\right)$ and thus $\bar{x} \in \Omega$. Therefore $\bar{x} \in \Gamma=\Omega \cap F(S)$. 
We now show that $\left\{x_{n}\right\}$ converges strongly to $z=P_{\Gamma}(I-D+\xi f)(z)$ which is the unique solution of the variational inequality (24). To do this, we first prove that $\lim \sup _{n \rightarrow \infty}\left\langle(D-\xi f) z, z-x_{n}\right\rangle \leq 0$. Choose a subsequence $\left\{x_{n_{j}}\right\}$ of $\left\{x_{n}\right\}$ such that

$$
\lim \sup \left\langle(D-\xi f) z, z-x_{n}\right\rangle=\lim _{j \rightarrow \infty}\left\langle(D-\xi f) z, z-x_{n_{j}}\right\rangle .
$$

Since $x_{n_{j}} \rightarrow \bar{x}$, we get

$$
\begin{aligned}
\lim \sup \left\langle(D-\xi f) z, z-x_{n}\right\rangle & =\lim _{j \rightarrow \infty}\left\langle(D-\xi f) z, z-x_{n_{j}}\right\rangle \\
& =\langle(D-\xi f) z, z-\bar{x}\rangle \leq 0 .
\end{aligned}
$$

Now from (27), we have

$$
\begin{aligned}
\left\|x_{n+1}-z\right\|^{2} & \leq\left(1-\alpha_{n} \bar{\gamma}\right)^{2}\left[\left\|x_{n}-z\right\|^{2}+2 \alpha^{4} N_{2}\right]+2 \alpha_{n} \xi \beta\left\|x_{n}-z\right\| \cdot\left\|x_{n+1}-z\right\|+2 \alpha_{n}\left\langle\xi f(z)-D z, x_{n+1}-z\right\rangle \\
& \leq\left(1-\alpha_{n} \bar{\gamma}\right)^{2}\left\|x_{n}-z\right\|^{2}+\alpha_{n} \xi \beta\left(\left\|x_{n}-z\right\|^{2}+\left\|x_{n+1}-z\right\|^{2}\right)+2 \alpha_{n}\left\langle\xi f(z)-D z, x_{n+1}-z\right\rangle+2 \alpha_{n}^{4} N_{2} \\
& \leq\left(1-\alpha_{n} \bar{\gamma}\right)\left\|x_{n}-z\right\|^{2}+\alpha_{n} \xi \beta\left(\left\|x_{n}-z\right\|^{2}+\left\|x_{n+1}-z\right\|^{2}\right)+2 \alpha_{n}\left\langle\xi f(z)-D z, x_{n+1}-z\right\rangle+2 \alpha_{n}^{4} N_{2} \\
& \leq\left(1-\frac{\alpha_{n}(\bar{\gamma}-\xi \beta)}{1-\alpha_{n} \xi \beta}\right)\left\|x_{n}-z\right\|^{2}+\frac{2 \alpha_{n}}{1-\alpha_{n} \xi \beta}\left(\left\langle\xi f(z)-D z, x_{n+1}-z\right\rangle+\alpha_{n}^{3} N_{2}\right) \\
& =\left(1-v_{n}\right)\left\|x_{n}-z\right\|+v_{n} \delta_{n},
\end{aligned}
$$

where

$$
v_{n}=\frac{\alpha_{n}(\bar{\gamma}-\xi \beta)}{1-\alpha_{n} \xi \beta} \quad \text { and } \quad \delta_{n}=\frac{2}{\bar{\gamma}-\xi \beta}\left[\left\langle\xi f(z)-D z, x_{n+1}-z\right\rangle+\alpha_{n}^{3} N_{2}\right] .
$$

It is easy to verify that $\sum_{n=0}^{\infty} v_{n}=\infty$ and $\lim _{\sup _{n \rightarrow \infty}} \delta_{n} \leq 0$. Therefore, from Lemma 2.5, we get $\left\|x_{n}-z\right\| \rightarrow 0$, as $n \rightarrow \infty$ and hence $\left\{x_{n}\right\}$ converges strongly to $z$. From (46) and (47), it is easy to see that $\left\{y_{n}\right\}$ and $\left\{z_{n}\right\}$ converge strongly $z$.

Case II: Assume that $\left\{\left\|x_{n}-p\right\|\right\}$ is not monotonically decreasing. For all $n \geq n_{0}$ (for some $n_{0}$ large enough), let $\tau: \mathbb{N} \rightarrow \mathbb{N}$ be defined by

$$
\tau(n)=\max \left\{k \in \mathbb{N}: k \leq n: \tau_{k} \leq \tau_{k+1}\right\} .
$$

Clearly, $\tau$ is nondecreasing since $\tau(n) \rightarrow \infty$ as $n \rightarrow \infty$ and

$$
0 \leq\left\|\chi_{\tau(n)}-p\right\| \leq\left\|x_{\tau(n)+1}-p\right\|, \quad \forall n \geq n_{0} .
$$

Following a similar argument as in Case I, we have $\left\|\left(I-T_{r_{\tau(n)}}^{\Theta_{2}}\right) A x_{\tau(n)}\right\| \rightarrow 0,\left\|S z_{\tau(n)}-z_{\tau(n)}\right\| \rightarrow 0$, and $\left\|x_{\tau(n)+1}-x_{\tau(n)}\right\| \rightarrow 0$. Also, we obtain

$$
\limsup _{n \rightarrow \infty}\left\langle(D-\xi f) p, p-x_{\tau(n)}\right\rangle \leq 0 .
$$

Now, since $\left\{x_{\tau(n)}\right\}$ is bounded, there exists a subsequence of $\left\{x_{\tau(n)}\right\}$ denoted by $\left\{x_{\tau\left(n_{j}\right)}\right\}$ which converges weakly to $\bar{x}$. Suppose $\left\{x_{\tau\left(n_{j}\right)}\right\}$ is such that

$$
\limsup _{n \rightarrow \infty}\left\langle\xi f(p)-D p, x_{\tau(n)+1}-p\right\rangle=\lim _{j \rightarrow \infty}\left\langle\xi f(p)-D p, x_{\tau\left(n_{j}\right)+1}-p\right\rangle .
$$

Since $x_{\tau(n)} \rightarrow \bar{x}$, and from (24), we have

$$
\begin{aligned}
\limsup _{n \rightarrow \infty}\left\langle\xi f(p)-D p, x_{\tau(n)+1}-p\right\rangle & =\lim _{j \rightarrow \infty}\left\langle\xi f(p)-D p, x_{\tau\left(n_{j}\right)+1}-p\right\rangle \\
& =\langle\xi f(p)-D p, \bar{x}-p\rangle \leq 0 .
\end{aligned}
$$

Therefore

$$
\limsup _{n \rightarrow \infty}\left\langle\xi f(p)-D p, x_{\tau(n)+1}-p\right\rangle \leq 0
$$


Similarly, as in (52) we obtain

$$
\begin{aligned}
\left\|x_{\tau(n)+1}-p\right\|^{2} \leq & \left(1-\alpha_{\tau(n)} \bar{\gamma}\right)^{2}\left[\left\|x_{\tau(n)}-p\right\|^{2}+2 \alpha^{4} N_{2}\right]+2 \alpha_{\tau(n)} \xi \beta\left\|x_{\tau(n)}-p\right\| \cdot\left\|x_{\tau(n)+1}-p\right\| \\
& +2 \alpha_{\tau(n)}\left\langle\xi f(p)-D p, x_{\tau(n)+1}-p\right\rangle \\
\leq & \left(1-\frac{\alpha_{\tau(n)}(\bar{\gamma}-\xi \beta)}{1-\alpha_{\tau(n)} \xi \beta}\right)\left\|x_{\tau(n)}-p\right\|^{2}+\frac{2 \alpha_{\tau(n)}}{1-\alpha_{\tau(n)} \xi \beta}\left[\left\langle\xi f(p)-D p, x_{\tau(n)+1}-p\right\rangle+\alpha_{n}^{3} N_{2}\right] .
\end{aligned}
$$

Since $\left\|x_{\tau(n)}-p\right\|^{2} \leq\left\|x_{\tau(n)+1}-p\right\|^{2}$, then from (54), we have

$$
\begin{aligned}
0 & \leq\left\|x_{\tau(n)+1}-p\right\|^{2}-\left\|x_{\tau(n)}-p\right\|^{2} \\
& \leq\left(1-\frac{\alpha_{\tau(n)}(\bar{\gamma}-\xi \beta)}{1-\alpha_{\tau(n)} \xi \beta}\right)\left\|x_{\tau(n)}-p\right\|^{2}+\frac{2 \alpha_{\tau(n)}}{1-\alpha_{\tau(n)} \xi \beta}\left[\left\langle\xi f(p)-D p, x_{\tau(n)+1}-p\right\rangle+\alpha_{n}^{3} N_{2}\right]-\left\|x_{\tau(n)}-p\right\|^{2} .
\end{aligned}
$$

It follows that

$$
\frac{\bar{\gamma}-\xi \beta}{1-\alpha_{\tau(n)} \xi \beta}\left\|x_{\tau(n)}-p\right\|^{2} \leq \frac{2}{1-\alpha_{\tau(n)} \xi \beta}\left[\left\langle\xi f(p)-D p, x_{\tau(n)+1}-p\right\rangle+\alpha_{n}^{3} N_{2}\right] .
$$

Since $\alpha_{\tau(n)} \rightarrow 0$, as $n \rightarrow \infty$ and from (53), we have

$$
\lim _{n \rightarrow \infty}\left\|x_{\tau(n)}-p\right\|=0 .
$$

As a consequence, we obtain for all $n \geq n_{0}$,

$$
0 \leq\left\|x_{n}-p\right\|^{2} \leq \max \left\{\left\|x_{\tau(n)}-p\right\|^{2},\left\|x_{\tau(n)+1}-p\right\|^{2}\right\}=\left\|x_{\tau(n)+1}-p\right\|^{2} .
$$

Hence, $\lim _{n \rightarrow \infty}\left\|x_{n}-p\right\|=0$. This implies that $\left\{x_{n}\right\}$ converges strongly to $p$. This complete the proof.

We now give the following consequences of Theorem 3.6.

1. Consider the following split mixed equilibrium problem. Find $x^{\star} \in C$ such that

$$
\left\{\begin{array}{l}
\Theta_{1}\left(x^{\star}, x\right)+\phi(x)-\phi\left(x^{\star}\right) \geq 0, \quad \forall x \in C, \\
\text { with } \\
y^{\star}=A x^{\star} \quad \text { which solves } \Theta_{2}\left(y^{\star}, y\right)+\varphi\left(y^{\star}\right)-\varphi(y) \geq 0, \quad \forall y \in Q .
\end{array}\right.
$$

The set of solutions of (57) is denoted by $\operatorname{MEP}\left(\Theta_{1}, \Theta_{2}, \phi, \varphi\right)$. In [44], the authors proved a weak convergence theorem for solving (57) and a fixed point problem of a nonlinear multi-valued mapping in real Hilbert spaces. Putting $h_{1}=h_{2}=0$ in Theorem 3.6, we obtain a strong convergence result for approximating a common solution of (57) and a fixed point problem for nonspreading mappings without prior knowledge of the operator norm in real Hilbert spaces. Thus, the following result complements the result in [44].

Corollary 3.7. Let $C$ and $Q$ be nonempty closed and convex subsets of real Hilbert spaces $H_{1}$ and $H_{2}$, respectively, and let $A: H_{1} \rightarrow H_{2}$ be a bounded linear operator. Let $\Theta_{1}: C \times C \rightarrow \mathbb{R}$ and $\Theta_{2}: Q \times Q \rightarrow \mathbb{R}$ be bifunctions which satisfy Assumption 2.3. Let $\phi: C \rightarrow \mathbb{R} \cup\{+\infty\}$ and $\varphi: Q \rightarrow \mathbb{R} \cup\{+\infty\}$ be proper, lowersemicontinuous and convex functions, and let $S: C \rightarrow C$ be a nonspreading mapping such that $F(S) \neq \emptyset$. Let $f: H_{1} \rightarrow H_{1}$ be a contraction mapping with constant $\beta \in(0,1)$ and let $D$ be a bounded linear operator with coefficient $\bar{\gamma} \in(0,1)$ such that $0<\xi<\frac{\bar{\gamma}}{\beta}$. Choose an initial guess $x_{1} \in H_{1}$ arbitrarily and let $\alpha_{n} \in[0,1], \beta_{n} \in[0,1], w_{n} \in(0,1)$, $r_{n}>0$ and $\lambda>0$. Assume that the nth iterate has been constructed, and set $m_{1}=\frac{\gamma_{1} A^{*}\left(T_{r_{1}}^{\theta_{2}}-I\right) A x_{1}}{\lambda}$. We then compute the $(n+1)$ th iterate via the formula

$$
\left\{\begin{array}{l}
m_{n+1}=\frac{\gamma_{n} A^{\star}\left(T_{r_{n}}^{\Theta_{2}}-I\right) A x_{n}}{\lambda}+\beta_{n} m_{n}, \\
y_{n}=x_{n}+\lambda m_{n+1}, \\
z_{n}=T_{r_{n}}^{\Theta_{1}} y_{n}, \\
x_{n+1}=\alpha_{n} \xi f\left(x_{n}\right)+\left(1-\alpha_{n} D\right)\left[\left(1-w_{n}\right) z_{n}+w_{n} S z_{n}\right],
\end{array}\right.
$$


for $n \geq 1$, where $A^{*}$ is the adjoint operator of A. Further, we choose the stepsize $\gamma_{n}$ such that, if

$$
n \in O:=\left\{n:\left(I-T_{r_{n}}^{\Theta_{2}}\right) A x_{n} \neq 0\right\},
$$

then

$$
\gamma_{n} \in\left(0, \frac{2\left\|\left(I-T_{r_{n}}^{\Theta_{2}}\right) x_{n}\right\|^{2}}{\left\|A^{\star}\left(I-T_{r_{n}}^{\Theta_{2}}\right) A x_{n}\right\|^{2}}\right), \quad \forall n \in O
$$

Otherwise, $\gamma_{n}=\gamma(\gamma$ being any nonnegative value $)$. Suppose $\Gamma:=M E P\left(\Theta_{1}, \Theta_{2}, \phi, \varphi\right) \cap F(S) \neq \emptyset$, and that Assumption 3.4 and the following condition is satisfied:

$$
\liminf _{n \rightarrow \infty} r_{n}>0 \text {. }
$$

Then, $\left\{x_{n}\right\}$ converges strongly to a point $z$, where $z=P_{\Gamma}(I-D+\xi f)(z)$ is a unique solution of

$$
\langle(D-\xi f) z, z-x\rangle \leq 0, \quad x \in \Gamma .
$$

2. In [38], Suantai et.al. proved a weak convergence result for finding a common solution of Problem (9) and a fixed point problem of a $\frac{1}{2}$-nonspreading multi-valued mapping in real Hilbert space.

Putting $h_{1}=h_{2}=\phi=\varphi=0$ in Theorem 3.6, we obtain a strong convergence result for approximating a common solution of (9) and a fixed point of a nonspreading mappings without prior knowledge of the operator norm. Thus, the following result complements the result of Suantai et.al. [38].

Corollary 3.8. Let $C$ and $Q$ be nonempty closed and convex subsets of real Hilbert spaces $H_{1}$ and $H_{2}$, respectively, and let $A: H_{1} \rightarrow H_{2}$ be a bounded linear operator. Let $\Theta_{1}: C \times C \rightarrow \mathbb{R}$ and $\Theta_{2}: Q \times Q \rightarrow \mathbb{R}$ be bifunctions which satisfy Assumption 2.3. Let $S: C \rightarrow C$ be a nonspreading mapping such that $F(S) \neq \emptyset$. Let $f: H_{1} \rightarrow H_{1}$ be a contraction mapping with constant $\beta \in(0,1)$, and let $D$ be a bounded linear operator with coefficient $\bar{\gamma} \in(0,1)$ such that $0<\xi<\frac{\bar{\gamma}}{\beta}$. Choose an initial guess $x_{1} \in H_{1}$ arbitrarily and let $\alpha_{n} \in[0,1], \beta_{n} \in[0,1]$, $w_{n} \in(0,1), r_{n}>0$ and $\lambda>0$. Assume that the $n$th iterate has been constructed, and set $m_{1}=\frac{\gamma_{1} A^{*}\left(T_{r_{1}}^{\theta_{2}}-I\right) A x_{1}}{\lambda}$. We then compute the $(n+1)$ th iterate via the formula

$$
\left\{\begin{array}{l}
m_{n+1}=\frac{\gamma_{n} A^{\star}\left(T_{r_{n}}^{\Theta_{2}}-I\right) A x_{n}}{\lambda}+\beta_{n} m_{n}, \\
y_{n}=x_{n}+\lambda m_{n+1}, \\
z_{n}=T_{r_{n}}^{\Theta_{1}} y_{n}, \\
x_{n+1}=\alpha_{n} \xi f\left(x_{n}\right)+\left(1-\alpha_{n} D\right)\left[\left(1-w_{n}\right) z_{n}+w_{n} S z_{n}\right],
\end{array}\right.
$$

for $n \geq 1$, where $A^{*}$ is the adjoint operator of A. Further, we choose the stepsize $\gamma_{n}$ such that, if

$$
n \in O:=\left\{n:\left(I-T_{r_{n}}^{\Theta_{2}}\right) A x_{n} \neq 0\right\},
$$

then

$$
\gamma_{n} \in\left(0, \frac{2\left\|\left(I-T_{r_{n}}^{\Theta_{2}}\right) x_{n}\right\|^{2}}{\left\|A^{\star}\left(I-T_{r_{n}}^{\Theta_{2}}\right) A x_{n}\right\|^{2}}\right), \quad \forall n \in O .
$$

Otherwise, $\gamma_{n}=\gamma(\gamma$ being any nonnegative value $)$. Suppose $\Gamma:=\operatorname{SEP}\left(\Theta_{1}, \Theta_{2}\right) \cap F(S) \neq \emptyset$, Assumption 3.4 is satisfied, and further suppose that the following condition is satisfied:

$$
\liminf _{n \rightarrow \infty} r_{n}>0 .
$$

Then, $\left\{x_{n}\right\}$ converges strongly to a point $z$, where $z=P_{\Gamma}(I-D+\xi f)(z)$ is a unique solution of

$$
\langle(D-\xi f) z, z-x\rangle \leq 0, \quad x \in \Gamma .
$$


3. Let $S: C \rightarrow C$ be a nonexpansive mapping in Theorem 3.6, then we have the following result:

Corollary 3.9. Let $C$ and $Q$ be nonempty closed and convex subsets of real Hilbert spaces $H_{1}$ and $H_{2}$, respectively, and let $A: H_{1} \rightarrow H_{2}$ be a bounded linear operator. Let $\Theta_{1}: C \times C \rightarrow \mathbb{R}$ and $\Theta_{2}: Q \times Q \rightarrow \mathbb{R}$ be bifunctions which satisfy Assumption 2.3. Let $h_{1}: C \rightarrow H_{1}$ and $h_{2}: Q \rightarrow H_{2}$ be $\theta_{1}, \theta_{2}$-inverse strongly monotone mappings respectively such that $\theta=\max \left\{\theta_{1}, \theta_{2}\right\}$. Let $\phi: C \rightarrow \mathbb{R} \cup\{+\infty\}$ and $\varphi: Q \rightarrow \mathbb{R} \cup\{+\infty\}$ be proper, lowersemicontinuous and convex functions, and let $S: C \rightarrow C$ be a nonexpansive mapping such that $F(S) \neq \emptyset$. Let $f: H_{1} \rightarrow H_{1}$ be a contraction mapping with constant $\beta \in(0,1)$, and let $D$ be a bounded linear operator with coefficient $\bar{\gamma} \in(0,1)$ such that $0<\xi<\frac{\bar{\gamma}}{\beta}$. Choose an initial guess $x_{1} \in H_{1}$ arbitrarily and let $\alpha_{n} \in[0,1]$, $\beta_{n} \in[0,1], w_{n} \in(0,1), r_{n} \in(0,2 \theta)$ and $\lambda>0$. Assume that the $n$th iterate has been constructed, and set $m_{1}=\frac{\gamma_{1} A^{*}\left(T_{r_{1}}^{\theta_{2}}-I\right) A x_{1}}{\lambda}$. We then compute the $(n+1)$ th iterate via the formula

$$
\left\{\begin{array}{l}
m_{n+1}=\frac{\gamma_{n} A^{\star}\left(T_{r_{n}}^{\Theta_{2}}-I\right) A x_{n}}{\lambda}+\beta_{n} m_{n}, \\
y_{n}=x_{n}+\lambda m_{n+1}, \\
z_{n}=T_{r_{n}}^{\Theta_{1}}\left(I-r_{n} h_{1}\right) y_{n}, \\
x_{n+1}=\alpha_{n} \xi f\left(x_{n}\right)+\left(1-\alpha_{n} D\right)\left[\left(1-w_{n}\right) z_{n}+w_{n} S z_{n}\right],
\end{array}\right.
$$

for $n \geq 1$, where $A^{*}$ is the adjoint operator of A. Further, we choose the stepsize $\gamma_{n}$ such that, if

$$
n \in O:=\left\{n:\left(I-T_{r_{n}}^{\Theta_{2}}\right) A x_{n} \neq 0\right\},
$$

then

$$
\gamma_{n} \in\left(0, \frac{2\left\|\left(I-T_{r_{n}}^{\Theta_{2}}\right) x_{n}\right\|^{2}}{\left\|A^{\star}\left(I-T_{r_{n}}^{\Theta_{2}}\right) A x_{n}\right\|^{2}}\right), \quad \forall n \in O .
$$

Otherwise, $\gamma_{n}=\gamma(\gamma$ being any nonnegative value). Suppose $\Gamma:=\Omega \cap F(S) \neq \emptyset$, Assumption 3.4 and the following conditions are satisfied:

(i) $\liminf _{n \rightarrow \infty} r_{n}>0$;

(ii) $0<\liminf _{n \rightarrow \infty} w_{n} \leq \lim \sup _{n \rightarrow \infty} w_{n}<1$.

Then $\left\{x_{n}\right\}$ converges strongly to a point $z$, where $z=P_{\Gamma}(I-D+\xi f)(z)$ is a unique solution of

$$
\langle(D-\xi f) z, z-x\rangle \leq 0, \quad x \in \Gamma .
$$

4. Putting $\xi=1$ and $D=I$ where $I$ is an identity mapping in Theorem 3.6, we have the following result:

Corollary 3.10. Let $C$ and $Q$ be nonempty closed and convex subsets of real Hilbert spaces $H_{1}$ and $H_{2}$, respectively, and let $A: H_{1} \rightarrow H_{2}$ be a bounded linear operator. Let $\Theta_{1}: C \times C \rightarrow \mathbb{R}$ and $\Theta_{2}: Q \times Q \rightarrow \mathbb{R}$ be bifunctions which satisfy Assumption 2.3. Let $h_{1}: C \rightarrow H_{1}$ and $h_{2}: Q \rightarrow H_{2}$ be $\theta_{1}, \theta_{2}$-inverse strongly monotone mappings respectively such that $\theta=\max \left\{\theta_{1}, \theta_{2}\right\}$. Let $\phi: C \rightarrow \mathbb{R} \cup\{+\infty\}$ and $\varphi: Q \rightarrow \mathbb{R} \cup\{+\infty\}$ be proper, lowersemicontinuous and convex functions, and let $S: C \rightarrow C$ be a nonspreading mapping such that $F(S) \neq \emptyset$. Let $f: H_{1} \rightarrow H_{1}$ be a contraction mapping with constant $\beta \in(0,1)$. Choose an initial guess $x_{1} \in H_{1}$ arbitrarily and let $\alpha_{n} \in[0,1], \beta_{n} \in[0,1], w_{n} \in(0,1), r_{n} \in(0,2 \theta)$ and $\lambda>0$. Assume that the $n$th iterate has been constructed, and set $m_{1}=\frac{\gamma_{1} A^{*}\left(T_{r_{1}}^{\theta_{2}}-I\right) A x_{1}}{\lambda}$. We then compute the $(n+1)$ th iterate via the formula

$$
\left\{\begin{array}{l}
m_{n+1}=\frac{\gamma_{n} A^{*}\left(T_{r_{n}}^{\Theta_{2}}-I\right) A x_{n}}{\lambda}+\beta_{n} m_{n}, \\
y_{n}=x_{n}+\lambda m_{n+1}, \\
z_{n}=T_{r_{n}}^{\Theta_{1}}\left(I-r_{n} h_{1}\right) y_{n}, \\
x_{n+1}=\alpha_{n} f\left(x_{n}\right)+\left(1-\alpha_{n}\right)\left[\left(1-w_{n}\right) z_{n}+w_{n} S z_{n}\right],
\end{array}\right.
$$

for $n \geq 1$, where $A^{*}$ is the adjoint operator of A. Further, we choose the stepsize $\gamma_{n}$ such that, if

$$
n \in O:=\left\{n:\left(I-T_{r_{n}}^{\Theta_{2}}\right) A x_{n} \neq 0\right\},
$$


then

$$
\gamma_{n} \in\left(0, \frac{2\left\|\left(I-T_{r_{n}}^{\Theta_{2}}\right) x_{n}\right\|^{2}}{\left\|A^{\star}\left(I-T_{r_{n}}^{\Theta_{2}}\right) A x_{n}\right\|^{2}}\right), \quad \forall n \in O .
$$

Otherwise, $\gamma_{n}=\gamma(\gamma$ being any nonnegative value). Suppose $\Gamma:=\Omega \cap F(S) \neq \emptyset$, Assumption 3.4 and the following conditions are satisfied:

(i) $\liminf _{n \rightarrow \infty} r_{n}>0$;

(ii) $0<\liminf _{n \rightarrow \infty} w_{n} \leq \lim \sup _{n \rightarrow \infty} w_{n}<1$;

then, $\left\{x_{n}\right\}$ converges strongly to a point $z$, where $z=P_{\Gamma}(f) z$ is a unique solution of

$$
\langle(I-f) z, z-x\rangle \leq 0, \quad x \in \Gamma .
$$

Remark 3.11. The condition that $\left\{\left(I-T_{r_{n}}^{\Theta_{2}}\right) A x_{n}\right\}$ is bounded is satisfied if the set of solutions $\Omega$ of SMEP (4) is bounded. If $\Omega$ is not bounded, then we need to verify the condition that $\left\{\left(I-T_{r_{n}}^{\Theta_{2}}\right) A x_{n}\right\}$ is bounded before applying our algorithm.

\section{Numerical example}

In this section, we provide a numerical result on the problem considered in Section 3.
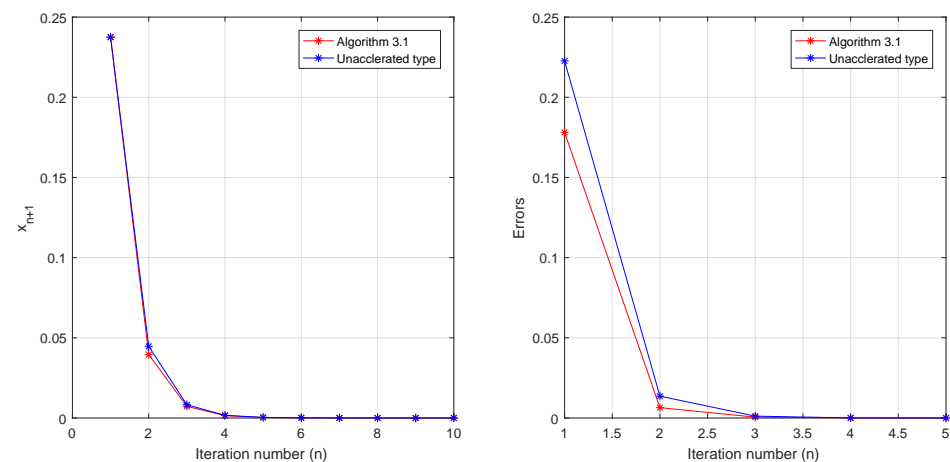

Figure 1: $x_{1}=0.2375$, Left: accuracy against number of iterations; Right: errors against numbers of iterations.

Example 4.1. Let $H_{1}=H_{2}=\mathbb{R}$ and $C=Q=[0,2]$. Define $\Theta_{1}: C \times C \rightarrow \mathbb{R}$ by $\Theta_{1}(x, y)=-\frac{1}{2} x^{2}+\frac{1}{2} y^{2}$, $h_{1}: C \rightarrow \mathbb{R}$ by $h_{1}(x)=x$ and $\phi: C \rightarrow \mathbb{R}$ by $\phi(x)=\frac{1}{2} x^{2}$. It is easy to see that

$$
T_{r_{n}}^{\Theta_{1}}(z)=\frac{z}{3 r_{n}+1}, \quad \forall z \in \mathbb{R} .
$$

Also, let $\Theta_{2}: \mathbb{R} \times \mathbb{R} \rightarrow \mathbb{R}$ be defined by $\Theta_{2}(u, v)=-3 u^{2}+2 u v+v^{2}, h_{2}: Q \rightarrow \mathbb{R}$ be defined by $h_{2}(u)=2 u$ and $\varphi: \mathbb{R} \rightarrow \mathbb{R}$ be defined by $\varphi(u)=u^{2}$, then

$$
T_{r_{n}}^{\Theta_{2}}(w)=\frac{w}{6 r_{n}+1}, \quad \forall w \in \mathbb{R} .
$$

Let $A: \mathbb{R} \rightarrow \mathbb{R}$ be defined by $A(x)=2 x$ for all $x \in \mathbb{R}$. Then $A$ is a bounded linear operator and $A^{T}(x)=2 x$ for all $x \in \mathbb{R}$. Clearly, $\Omega:=\left\{p \in G M E P\left(\Theta_{1}, h_{1}, \phi\right): A p \in G M E P\left(\Theta_{2}, h_{2}, \varphi\right)\right\}=\{0\}$. This shows that $\Omega$ is bounded and thus, the sequence $\left\{\left(I-T_{r_{n}}^{\Theta_{2}}\right) A x_{n}\right\}$ is also bounded. 

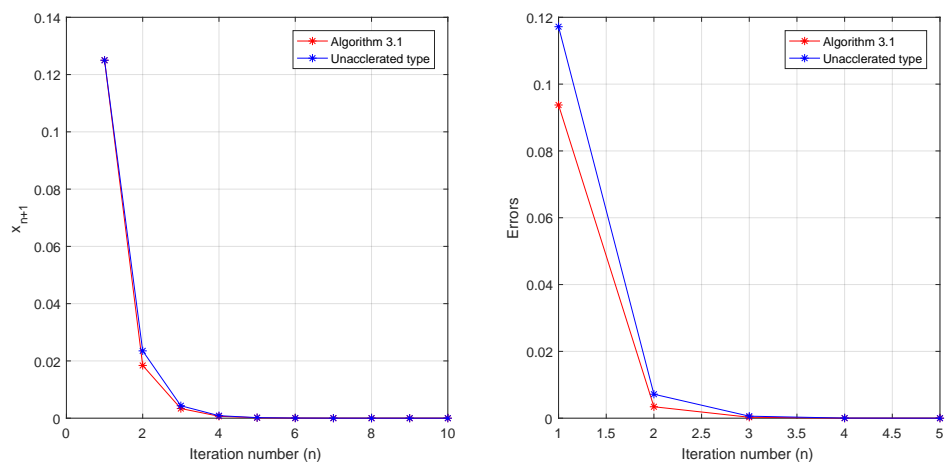

Figure 2: $x_{1}=0.1250$, Left: accuracy against number of iterations; Right: errors against numbers of iterations.
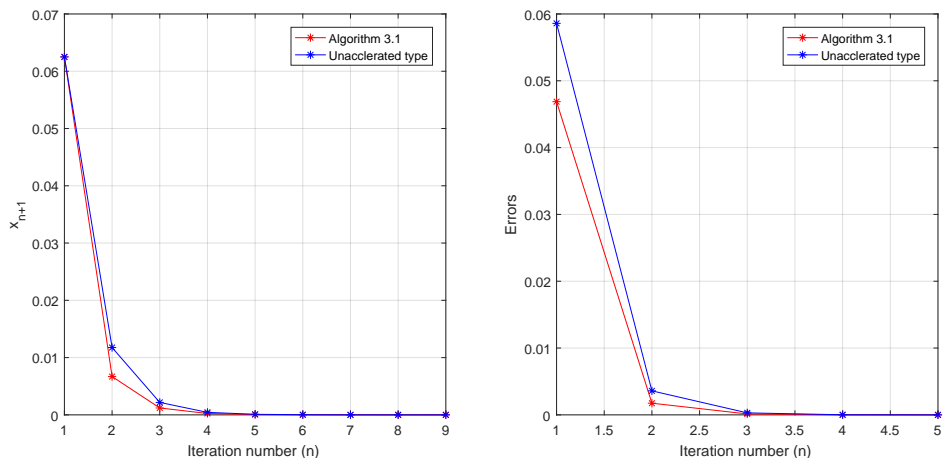

Figure 3: $x_{1}=0.0625$, Left: accuracy against number of iterations; Right: errors against numbers of iterations.
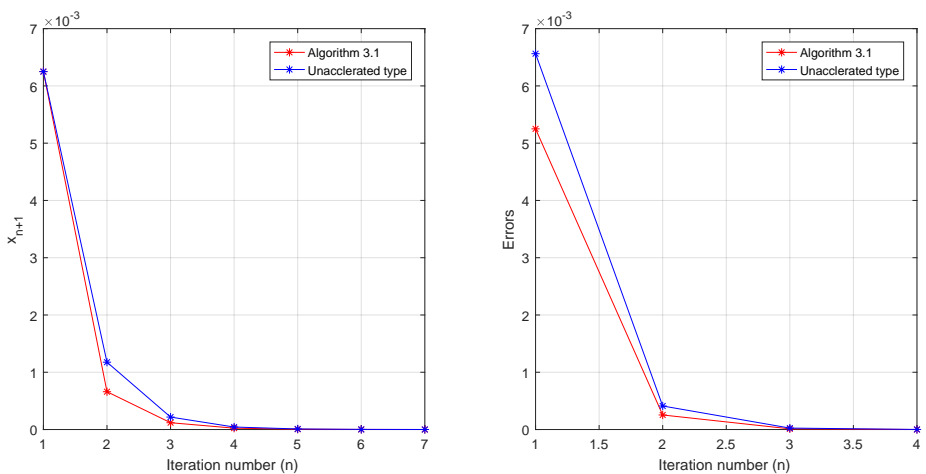

Figure 4: $x_{1}=0.007$, Left: accuracy against number of iterations; Right: errors against numbers of iterations.

Define $S: \mathbb{R} \rightarrow \mathbb{R}$ by

$$
S x= \begin{cases}x, & \text { if } x \in(-\infty, 1), \\ 1, & \text { if } x \in[1,+\infty) .\end{cases}
$$

It is easy to see that $S$ is nonspreading and $\Gamma=\{0\}$. Take $\xi=1, D=I$, where $I$ is an identity mapping and $f: \mathbb{R} \rightarrow \mathbb{R}$ be defined by $f(x)=\frac{x}{2}$. Choose $\alpha_{n}=\frac{1}{n+1}, w_{n}=\frac{1}{5\left(1+\frac{1}{n}\right)}, r_{n}=\frac{2}{n+1}, \beta_{n}=\frac{1}{2(n+1)^{4}}$ and $\lambda=1.5$, 
Table 1

\begin{tabular}{|c|c|c|}
\hline Initial values & Algorithm 3.1 & Unaccelerated alg. \\
\hline$x_{1}=0.0005$ & 0.0011 & 0.0027 \\
\hline$x_{1}=0.01$ & 0.0020 & 0.0262 \\
\hline$x_{1}=0.1$ & 0.0026 & 0.0357 \\
\hline$x_{1}=10$ & 0.0038 & 0.1119 \\
\hline
\end{tabular}

and set $m_{1}=\frac{\gamma_{1}}{1.5}\left(\frac{-24 r_{n}}{6 r_{n}+1}\right) x_{1}$. Then Algorithm 3.1 gives the following:

$$
\left\{\begin{array}{l}
m_{n+1}=\frac{\gamma_{n}}{1.5}\left(\frac{-24 r_{n}}{6 r_{n}+1}\right) x_{n}+\frac{m_{n}}{2(n+1)^{4}}, \\
y_{n}=x_{n}+1.5 m_{n+1}, \\
z_{n}=\frac{1}{3 r_{n}+1}\left(\frac{n-1}{n+1}\right) y_{n}, \\
x_{n+1}=\frac{1}{n+1} f\left(x_{n}\right)+\frac{n}{n+1}\left[\frac{4 n+5}{5(n+1)} z_{n}+\frac{n}{5(n+1)} S z_{n}\right], \quad n \geq 1 .
\end{array}\right.
$$

We now make a different choice of the initial value $x_{1}$ and use $\epsilon<10^{-6}$ for the stopping criterion.

Case 1: $x_{1}=0.2375, \quad$ Case 2: $x_{1}=0.1250, \quad$ Case 3: $x_{1}=0.0625, \quad$ Case 4: $x_{1}=0.007$.

We note that the choice of $\gamma_{n}$, as long as it is in the range, does not have any significant effect on either the number of iterations, nor the cpu time. Using Matlab version 2016b, we compare the computational result of Algorithm 3.1 with its unaccelerated form (i.e. taking $\beta_{n}=0$ ) and plot the graphs of accuracy against number of iterations, and errors against number of iterations (see Figure 1-4 and Table 1) which are located after the references below. This shows that Algorithm 3.1 converges faster and is more efficient than its unaccelerated form (i.e. when $\beta_{n}=0$ ).

\section{References}

[1] Blum E., From optimization and variational inequalities to equilibrium problems, Math. Student, 1994, 63(1-4), 123-145

[2] Ceng L.-C., Yao J.-C., A hybrid iterative scheme for mixed equilibrium problems and fixed point problems, J. Comput. Appl. Math., 2008, 214, 186-201

[3] Combettes P. L., Hirstoaga S. A., Equilibrium programming in Hilbert space, J. Nonlinear Convex Anal., 2005, 6, 117-136

[4] Flam S. D., Antipin A. S., Equilibrium programming using proximal-like algorithm, Math. Programming, 1997, 78(1), Ser. A, 29-41

[5] Censor Y., Eflving T., A multiprojection algorithm using Bregman projections in a product space, Numer. Algorithms, 1994, 8, 221-239

[6] Censor Y., Bortfeld T., Martin B., Trofimov A., A unified approach for inversion problems in intensity-modulated radiation therapy, Phys. Med. Biol., 2006, 51(2), 2353-2365 
[7] Censor Y., Elfving T., Kopf N., Bortfeld T., The multiple-set split feasibility problem and its application for inverse problems, Inverse Problem, 2005, 21(6), 2071-2084

[8] Censor Y., Motova A., Segal A., A pertubed projections and subgradient projections for the multiple-set split feasibility problem, J. Math. Anal. Appl., 2007, 327, 1244-1256

[9] Chen T., Shen J., Image processing and Analysis variational, PDE, Wavelent and Stochastic Methods, SIAM, Philadelpha, 2005

[10] Abass H. A., Ogbuisi F. U., Mewomo O. T., Common solution of split equilibrium problem and fixed point problem with no prior knowledge of operator norm, U.P.B. Sci. Bull., Series A, 2018, 80(1), 175-190

[11] Halpern B., Fixed points of nonexpanding maps, Bull. Amer. Math. Soc., 1967, 73, 957-961

[12] He S., Yeng C., Boundary point algorithms for minimum norm fixed points of nonexpansive mappings, Fixed Point Theory Appl., 2014, 56

[13] Jolaoso L. O., Ogbuisi F. U., Mewomo 0. T., An iterative method for solving minimization, variational inequality and fixed point problems in reflexive Banach spaces, Adv. Pure Appl. Math., 2017, DOI: 10.1515/apam-2017-0037

[14] Krasnoselskii M. A., Two remarks on the method of successive approximations, Usp. Math. Nauk., 1955, 10, 123-127

[15] Mann W. R., Mean value methods in iterations, Proc. Amer. Math. Soc., 1953, 4, 506-510

[16] Nakajo K., Takahashi W., Strong convergence theorems for nonexpansive mappings and nonexpansive semigroups, J. Math. Anal. Appl., 2003, 279(2), 372-379

[17] Ogbuisi F. U., Mewomo 0. T., Convergence analysis of common solution of certain nonlinear problems, Fixed Point Theory, 2018, 19(1), 335-358

[18] Mewomo O. T., Ogbuisi F. U., Convergence analysis of an iterative method for solving multiple-set split feasibility problems in certain Banach spaces, Quest. Math., 2018, 14(1), 129-148

[19] Ogbuisi F. U., Mewomo O. T., Iterative solution of split variational inclusion problem in a real Banach space, Afr. Mat., 2017, 28(1-2), 295-309

[20] Ogbuisi F. U., Mewomo O. T., On split generalized mixed equilibrium problems and fixed point problems with no prior knowledge of operator norm, J. Fixed Point Theory Appl., 2016, 19(3), 2109-2128

[21] Okeke C. C., Mewomo 0. T., On split equilibrim problem, variational inequality problem and fixed point problem for multivalued mappings, Ann. Acad. Rom. Sci. Ser. Math. Appl., 2017, 9(2), 255-280

[22] Shehu Y., Mewomo 0. T., Further investigation into split common fixed point problem for demicontractive operators, Acta Math. Sin. (Engl. Ser.), 2016, 32(11), 1357-1376

[23] Shehu Y., Mewomo O. T., Ogbuisi F. U., Further investigation into approximation of a common solution of fixed point problems and split feasibility problems, Acta Math. Sci. Ser. B (Engl. Ed.), 2016, 36(3), 913-930

[24] Moudafi A., Viscosity approximation method for fixed-points problems, J. Math. Anal. Appl., 2000, 241(1), 46-55

[25] Xu H. K., Viscosity approximation method for nonexpansive mappings, J. Math. Anal. Appl., 2004, 298(1), 279-291

[26] Polyak B. T., Some methods of speeding up the convergence of iteration methods, U.S.S.R. Comput. Math. Math. Phys., 1964, 4(5), 1-17

[27] Alvarez F., Attouch H., An inertial proximal method for monotone operators via discretization of a nonlinear oscillator with damping, Set-Valued Anal., 2001, 9(1-2), 3-11

[28] Moudafi A., Oliny M., Convergence of a splitting inertial proximal method for monotone operators, J. Comput. Appl. Math., 2003, 155(2), 447-454

[29] Lorenz D., Pock T., An inertial forward-backward algorithm for monotone inclusions, J. Math. Imaging Vision, 2015, 51(2), 311-325

[30] Chen C., Chan R. H., Ma S., Yang J., Inertial proximal ADMM for linearly constrained separable convex optimization, SIAM J. Imaging Sci., 2015, 8(4), 2239-2267

[31] Beck A., Teboulle M., A fast iterative shrinkage-thresholding algorithm for linear inverse problem, SIAM J. Imaging Sci., 2009, 2(1), 183-202

[32] Chambole A., Dossal C. H., On the convergence of the iterates of the "fast shrinkage/thresholding algorithm", J. Optim. Theory Appl., 2015, 166(3), 968-982

[33] Mainge P. E., Convergence theorems for inertial KM-type algorithms, J. Comput. Appl. Math., 2008, 219(1), 223-236

[34] Bot R. I., Csetnek E. R., Hendrich C., Inertial Douglas-Rachford splitting for monotone inclusions, Appl. Math. Comput., 2015, 256, 472-487

[35] Picard E., Memoire sur la theorie des equations aux derives partielles et la methode des approximation successive, J. Math. Pures et Appl., 1890, 6, 145-210

[36] Nocedal J., Wright S. J., Numerical Optimization, Spinger Series in Operations Research and Financial Engineering, Vol 2, 2nd Edition, Spinger, Berlin, 2006

[37] Dong Q. L., Yuan H. B., Accelerated Mann and CQ algorithms for finding a fixed point of nonexpansive mapping, Fixed Point Theory Appl., 2015, 2015:125

[38] Suntai S., Cholamjiak P., Cho Y. J., Cholamjiak W., On solving split equilibrium problems and fixed point problems of nonspreading multi-valued mappings in Hilbert space, Fixed Point Theory Appl., 2016, 2016:35

[39] Rizvi S. H., A strong convergence theorem for split mixed equilibrium and fixed point problems for nonexpansive mappings in Hilbert space, J. Fixed Point Thoery Appl., 2018, 20(8), DOI: 10.1007/s11784-018-0487-8 
[40] Hendrickx J. M., Olshevsky A., Matrix P-norms are NP-hard to approximate if $p \neq 1,2, \infty$, SIAM J. Matrix Anal. Appl., 2012, 31, 2802-2812

[41] Hussain N., Marino G., Abdou A. N., On Mann's method with viscosity for nonexpansive and nonspreading mapping in Hilbert spaces, Abstr. Appl. Anal., 2014, Article ID: 152530, DOI: 10.1155/2014/152530

[42] Li S., Li L., Cao L., He X., Yue X., Hybrid extragradient method for generalized mixed equilibrium problem and fixed point problems in Hilbert space, Fixed Point Theory Appl., 2013, 2013:240

[43] Xu H. K., Another control condition in an iterative method for nonexpansive mappings, Bull. Aust. Math. Soc., 2002, 65(1), 109-113

[44] Onjai-uea N., Phuengrattana W., On solving split mixed equilibrium problems and fixed point problems of hybrid-type multivalued mappings in Hilbert spaces, J. Ineq. Appl., 2017, 2017:137 